\title{
Fixed Switching Frequency Direct Model Predictive Control with Continuous and Discontinuous Modulation for Grid-Tied Converters with $L C L$ Filters
}

\author{
Petros Karamanakos, Senior Member, IEEE, Mehrdad Nahalparvari Student Member, IEEE, \\ and Tobias Geyer, Senior Member, IEEE
}

\begin{abstract}
This paper proposes two alternatives of a direct model predictive control (MPC) scheme for a three-phase twolevel grid-connected converter with an $L C L$ filter. Although both approaches are implemented as direct control methods, i.e., they combine control and modulation in one computational stage, they operate the converter at a constant switching frequency and generate a discrete grid current harmonic spectrum. To achieve this, the first method allows for one switching transition per phase and sampling interval, implying that a fixed modulation cycle akin to pulse width modulation (PWM) results. Moreover, by appropriately designing the objective function of the optimization problem underlying MPC, grid current distortions similar to those of space vector modulation (SVM) are produced. As for the second approach, two phases are allowed to switch per sampling interval, emulating the behavior of discontinuous PWM. Consequently, thanks to the introduced formulations, harmonic limitations imposed by relevant grid codes can be met with the proposed methods. Furthermore, owing to the multipleinput multiple-output (MIMO) nature of both approaches, all output variables of the system can be simultaneously controlled. Finally, the inherent full-state information of MPC renders an additional active damping loop unnecessary, further simplifying the controller design. The presented performance assessment highlights the potential benefits of both proposed MPC-based algorithms.
\end{abstract}

Index Terms-Model predictive control (MPC), optimal control, converter control, pulse width modulation (PWM), harmonics, MIMO control, active damping, grid-connected power converters.

\section{INTRODUCTION}

$\mathbf{C}$ ONTROL schemes based on model predictive control (MPC) [1] were introduced in the power electronics community as early as in the 1980 s, see, e.g., [2]. Nonetheless, MPC has not gained much attention before early 2000s. With the aid of ever-increasing computational power, however, this changed and a renewed interest was developed towards MPC. As a result, several MPC-based concepts, tailored to the needs of power electronic systems, have been introduced since then [3]. The published methods clearly highlight some of the

P. Karamanakos (corresponding author) is with the Faculty of Information Technology and Communication Sciences, Tampere University, 33101 Tampere, Finland; e-mail: p.karamanakos@ieee.org

M. Nahalparvari is with the School of Electrical Engineering and Computer Science, KTH Royal Institute of Technology, 10044 Stockholm, Sweden; email: mnah@kth.se

T. Geyer is with ABB System Drives, 5300 Turgi, Switzerland; e-mail: t.geyer@ieee.org inherent characteristics of MPC, such as, system constraint satisfaction (owing to the underlying constrained optimization problem) as well as its ability to handle multiple-input and multiple-output (MIMO) systems with complex, nonlinear dynamics, and integer manipulated variables.

MPC can be implemented as indirect or direct controller. Indirect MPC - according to which the control input is translated into the switching signals via a modulator-is most commonly implemented in its explicit MPC variant [4]-[7]. However, explicit MPC is computationally feasible only for systems with a low-dimensional state where the memory requirements are not significant. Moreover, it is ill suited to systems with timevarying references and parameters. Because of the above, explicit MPC is not favored in the power electronics community.

The most utilized MPC strategy - at least in the academic community - is direct MPC, also referred to as finite control set MPC (FCS-MPC) [3]. FCS-MPC, being a direct control strategy, i.e., the converter switches are directly manipulated [8], can achieve fast transients. Furthermore, it inherits the ease of implementation of direct controllers, such as direct torque/power control (DTC/DPC). On the other hand, FCSMPC suffers from two main drawbacks, namely, the pronounced computational complexity, and the variable switching frequency.

Regarding the former, since the optimization variable, i.e., the switch position, is modeled as an integer, the formulated optimization problem is a (mixed) integer program [9]. This means that its computational complexity increases exponentially with the number of candidate solutions, i.e., the sequences of switch positions. Although there exist some algorithms that decrease its average computational load [10], [11], the problem is typically solved by exhaustively enumerating the possible switching sequences [12], [13]. Moreover, given that the direct MPC problem has to be solved in real time within a matter of a few tens of microseconds, it can become computationally intractable.

As for the second disadvantage of direct MPC, a variable switching frequency leads to a non-discrete harmonic current/voltage spectrum [14]. For machine-connected converters such a spectrum is not relevant, thus direct MPC is a good choice. Grid-tied converters, however, have to meet harmonic grid codes at the point of common coupling (PCC) which impose stringent limits on even and non-integer harmonics. 
Therefore, direct MPC is, in general, not suitable for such applications [8, Section 11.2.5], [15, Section VIII].

Motivated by the above, some methods have been presented that deal with the issues of variable switching frequency and/or non-deterministic harmonic spectra. For example, deadbeatbased controllers were introduced in [16], [17], according to which the reference signals are fed into a modulator. By doing so, the switching frequency is kept constant and the output current harmonic spectrum is discrete. The presence of a modulator, however, slows down the fast transient responses inherent to direct control schemes. Moreover, such approaches are not applicable to MIMO systems. An alternative was proposed in [18]. Therein, MPC was augmented with a band-stop filter with the aim of harmonic spectrum shaping. Although the energy in undesired harmonics is reduced, a variable switching frequency-and thus non-deterministic switching power losses-results.

In a different direction, but with the same objectives as those aforementioned, works like [19]-[28] propose direct MPC schemes that allow the switch position to change not only at the discrete time instants, but also at any time instant within the sampling interval. To this aim, the notion of "duty cycle" is adopted, i.e., MPC computes the time instant within the sampling interval the new switch position should be applied to the converter. However, methods such as [20], [22]-[25], [27], are prone to suboptimality since the optimization problem is solved in two steps, i.e., the optimal switch positions are computed in the first optimization step, while the second step derives the "duty cycles". Furthermore, although the MPC algorithms in [19], [23], [26], [27] guarantee a constant switching frequency, they do not generate symmetrical switching patterns, resulting in non-discrete harmonic spectra. As for [21], [28], although optimality is ensured, a fixed switching frequency is not.

To tackle both problems of pronounced computational complexity and variable switching frequency, the notion of precomputed switching sequences [29]-[32] was utilized by direct MPC. The advantage of this is that the optimization variable is the switching instants of the switching sequence, which renders the optimization problem computationally tractable. However, these methods are either limited only to simple, single-output systems, such as dc-dc converters [32], or the optimization problem can be formulated only as an unconstrained one with respect to time [29]-[31]. As a result, the generated switching sequences are not necessarily symmetrical, and, consequently, the harmonic spectra are non-discrete.

To address the shortcomings of the methods discussed above, a direct MPC scheme for a variable speed drive system was proposed in [33]. This method manages to operate the converter at a fixed switching frequency as well as to produce discrete stator current harmonic spectrum. This is achieved by forcing each phase leg to switch once per sampling interval in a specific chronological order. This implies that a fixed modulation cycle is adopted akin to carrier-based pulse width modulation (CB-PWM) or space vector modulation (SVM) [34].

This paper employs the method introduced in [33] to control a three-phase two-level converter connected to the grid via an intermediate $L C L$ filter. The algorithm is refined and implemented as a MIMO approach to meet the multiple control objectives. To this aim, a linear approximation of the references of the controlled variables, a longer prediction horizon and a heavier penalization of the output error at the discrete time steps are implemented to improve the system performance. As a result, the grid current harmonics meet the limits specified by the IEEE 519 grid standard [35]. Moreover, although the controller operates the converter at a (fixed) switching frequency of almost twice the resonance frequency, an outer damping loop-which is most often necessary with conventional control techniques [36] - is not required thanks to the full-state control nature of MPC.

In addition, to further reduce the switching frequency while not violating the harmonic limitations and exciting the resonance frequency, a second formulation of the MPC problem is proposed that emulates the behavior of $120^{\circ}$ discontinuous PWM (DPWM) [34]. More specifically, the presented MPC algorithm-while utilizing the same refinements mentioned above-generates switching sequences similar to that of $120^{\circ}$ DPWM (also known as DPWMMIN) by clamping each phase leg to the lower dc rail for $1 / 3$ of the fundamental period. As a consequence, the controller can operate the converter at a switching frequency that is $33 \%$ lower than that of the MPC that emulates SVM. Finally, both MPC approaches, owing to their direct MPC nature, exhibit fast transient responses when changes in the power references occur.

This paper is structured as follows. Section II introduces the mathematical model of the case study used in this paper. The proposed MPC strategies that emulate SVM and DPWMMIN are presented and analyzed in Sections III and IV, respectively. In Section $\mathrm{V}$, the performance of the two algorithms is assessed. Conclusions are drawn in Section VI.

Throughout the paper, unless otherwise stated, the quantities are normalized and presented in the per unit (p.u.) system. The modeling of the system and the formulation of the control problem is done in the orthogonal $\alpha \beta$ reference frame. Thus, any variable $\boldsymbol{\xi}_{a b c}=\left[\begin{array}{lll}\xi_{a} & \xi_{b} & \xi_{c}\end{array}\right]^{T}$ in the three-phase ( $\left.a b c\right)$ system is transformed into a two-dimensional variable $\boldsymbol{\xi}_{\alpha \beta}=\left[\xi_{\alpha} \xi_{\beta}\right]^{T}$ in the stationary $(\alpha \beta)$ reference frame using the operation $\boldsymbol{\xi}_{\alpha \beta}=\boldsymbol{K} \boldsymbol{\xi}_{a b c}$, where $\boldsymbol{K}$ is the Clarke transformation matrix

$$
\boldsymbol{K}=\frac{2}{3}\left[\begin{array}{ccc}
1 & -\frac{1}{2} & -\frac{1}{2} \\
0 & \frac{\sqrt{3}}{2} & -\frac{\sqrt{3}}{2}
\end{array}\right] .
$$

Finally, variables in the $a b c$-plane are denoted with the corresponding subscript. For convenience, the subscript of the variables in the $\alpha \beta$-plane is omitted.

\section{Mathematical Model of the System}

The examined system, consisting of a three-phase two-level grid-connected converter with an $L C L$ filter, is shown in Fig. 1. The dc-link voltage is assumed to be constant and equal to its nominal value $V_{\mathrm{dc}}$. Furthermore, as it is common with grid-tied converters, an $L C L$ filter is chosen due to its stronger attenuation of harmonics beyond the resonance frequency, as compared, e.g., to an $L$ filter. In the following, the discrete-time state-space model of the system is derived 


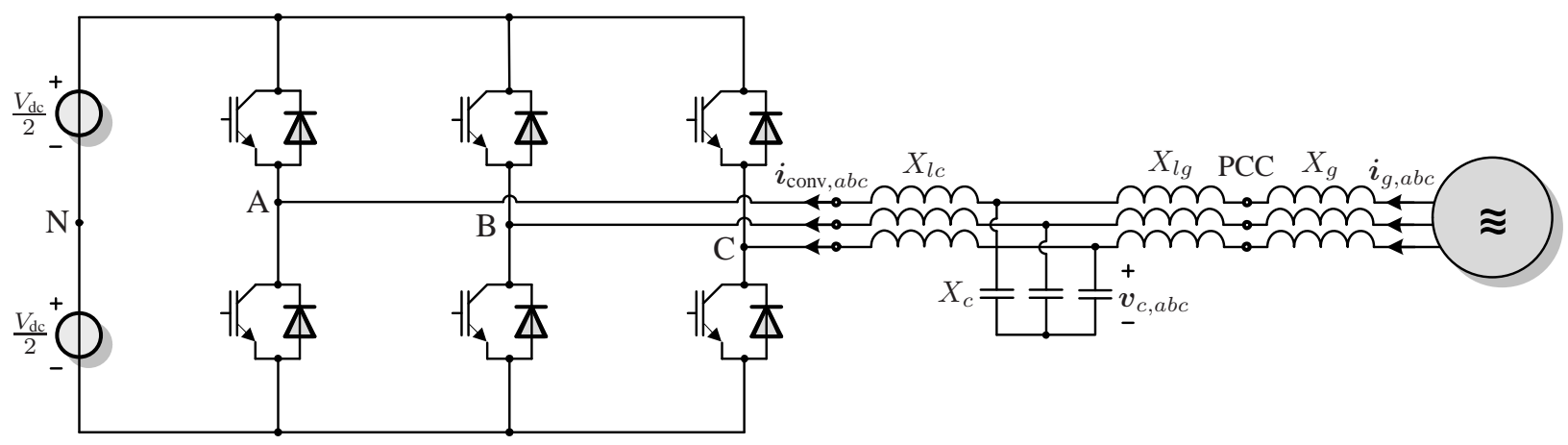

Fig. 1: Two-level grid-connected converter with an $L C L$ filter.

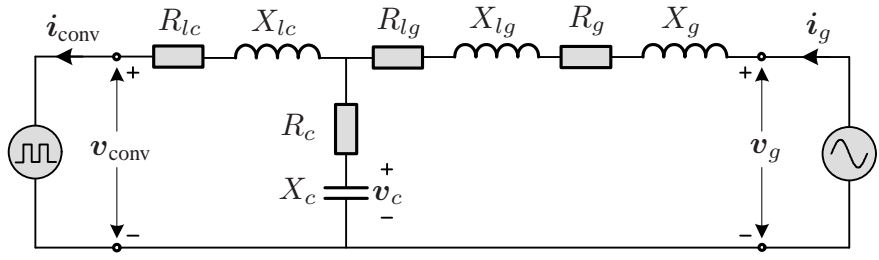

Fig. 2: Equivalent circuit of the grid-connected converter with an $L C L$ filter in the stationary $(\alpha \beta)$ frame.

that will serve as the internal prediction model for the proposed MPC methods.

Firstly, the converter model is derived. This can be seen as a gain. Specifically, each phase leg of the converter in phase $x \in\{a, b, c\}$ can assume two states $u_{x} \in \mathcal{U}=\{-1,1\}$. Consequently, the converter can produce two discrete voltage levels $-\frac{V_{\mathrm{dc}}}{2}$ and $\frac{V_{\mathrm{dc}}}{2}$, respectively [37]. Thus, the converter output voltage $\boldsymbol{v}_{\text {conv }}$ is given by

$$
\boldsymbol{v}_{\mathrm{conv}}=\frac{V_{\mathrm{dc}}}{2} \boldsymbol{K} \boldsymbol{u}_{a b c}
$$

with

$$
\boldsymbol{u}_{a b c}=\left[\begin{array}{lll}
u_{a} & u_{b} & u_{c}
\end{array}\right]^{T} \in \mathcal{U}^{3} .
$$

In a next step, the state-space model of the system is derived based on the differential equations that describe its dynamics. To this end, the equivalent circuit of the system, depicted in Fig. 2, is utilized. Moreover, as state variables are chosen the converter current $\boldsymbol{i}_{\text {conv }}$, grid current $\boldsymbol{i}_{g}$, capacitor voltage $\boldsymbol{v}_{c}$, and grid voltage $\boldsymbol{v}_{g}$. Thus, it follows that

$$
\begin{aligned}
\frac{\mathrm{d} \boldsymbol{i}_{\mathrm{conv}}}{\mathrm{d} t} & =\frac{1}{X_{l c}}\left(\boldsymbol{v}_{c}-\left(R_{l c}+R_{c}\right) \boldsymbol{i}_{\mathrm{conv}}+R_{c} \boldsymbol{i}_{g}-\boldsymbol{v}_{\mathrm{conv}}\right) \\
\frac{\mathrm{d} \boldsymbol{i}_{g}}{\mathrm{~d} t} & =\frac{1}{X_{g r}}\left(\boldsymbol{v}_{g}-\left(R_{g r}+R_{c}\right) \boldsymbol{i}_{g}+R_{c} \boldsymbol{i}_{\mathrm{conv}}-\boldsymbol{v}_{c}\right) \\
\frac{\mathrm{d} \boldsymbol{v}_{c}}{\mathrm{~d} t} & =\frac{1}{X_{c}}\left(\boldsymbol{i}_{g}-\boldsymbol{i}_{\mathrm{conv}}\right) \\
\frac{\mathrm{d} \boldsymbol{v}_{g}}{\mathrm{~d} t} & =\omega_{g}\left[\begin{array}{cc}
0 & -1 \\
1 & 0
\end{array}\right] \boldsymbol{v}_{g},
\end{aligned}
$$

where $R_{l c}$ and $X_{l c}$ are the converter-side filter resistance and reactance, respectively. $R_{g r}$ and $X_{g r}$ are the grid-side equivalent resistance and reactance, respectively, equal to $R_{g r}=R_{l g}+R_{g}$ and $X_{g r}=X_{l g}+X_{g}$, with $R_{l g}\left(X_{l g}\right)$ and $R_{g}$ $\left(X_{g}\right)$ being the grid-side filter and grid resistance (reactance), respectively. Moreover, $X_{c}$ and $R_{c}$ are the reactance and internal resistance of the filter capacitor, respectively. Finally, $\omega_{g}$ is the angular grid frequency.

Based on (4) the continuous-time state-space model is written as

$$
\begin{aligned}
\frac{\mathrm{d} \boldsymbol{x}(t)}{\mathrm{d} t} & =\boldsymbol{F} \boldsymbol{x}(t)+\boldsymbol{G} \boldsymbol{K} \boldsymbol{u}_{a b c}(t) \\
\boldsymbol{y}(t) & =\boldsymbol{C} \boldsymbol{x}(t),
\end{aligned}
$$

where, as mentioned above, the state vector is

$$
\boldsymbol{x}=\left[\begin{array}{llll}
\boldsymbol{i}_{\mathrm{conv}}^{T} & \boldsymbol{i}_{g}^{T} & \boldsymbol{v}_{c}^{T} & \boldsymbol{v}_{g}^{T}
\end{array}\right]^{T} \in \mathbb{R}^{8} .
$$

The control input is the three-phase switch position $\boldsymbol{u}_{a b c}$ as defined by (3). Moreover, the converter and grid current along with the capacitor voltage are the output variables, i.e., $\boldsymbol{y}=\left[\begin{array}{llll}\boldsymbol{i}_{\text {conv }}^{T} & \boldsymbol{i}_{g}^{T} & \boldsymbol{v}_{c}^{T}\end{array}\right]^{T} \in \mathbb{R}^{6}$. The dynamics $\boldsymbol{F}$, input $\boldsymbol{G}$ and output matrices $C$ are

$$
\begin{aligned}
& \boldsymbol{F}=\left[\begin{array}{cccc}
-\frac{R_{l c}+R_{c}}{X_{l c}} \boldsymbol{I}_{2} & \frac{R_{c}}{X_{l c}} \boldsymbol{I}_{2} & \frac{1}{X_{l c}} \boldsymbol{I}_{2} & \mathbf{0}_{2 \times 2} \\
\frac{R_{c}}{X_{g r}} \boldsymbol{I}_{2} & -\frac{R_{g r}+R_{c}}{X_{g r}} \boldsymbol{I}_{2} & -\frac{1}{X_{g r}} \boldsymbol{I}_{2} & \frac{1}{X_{g r}} \boldsymbol{I}_{2} \\
-\frac{1}{X_{c}} \boldsymbol{I}_{2} & \frac{1}{X_{c}} \boldsymbol{I}_{2} & \mathbf{0}_{2 \times 2} & \mathbf{0}_{2 \times 2} \\
\mathbf{0}_{2 \times 2} & \mathbf{0}_{2 \times 2} & \mathbf{0}_{2 \times 2} & \omega_{g}\left[\begin{array}{cc}
0 & -1 \\
1 & 0
\end{array}\right]
\end{array}\right] \\
& \boldsymbol{G}=-\frac{V_{\mathrm{dc}}}{2 X_{l c}}\left[\begin{array}{ll}
\boldsymbol{I}_{2} & \mathbf{0}_{2 \times 6}
\end{array}\right]^{T}, \text { and } \boldsymbol{C}=\left[\begin{array}{ll}
\boldsymbol{I}_{6} & \mathbf{0}_{6 \times 2}
\end{array}\right],
\end{aligned}
$$

where $\boldsymbol{I}$ and $\mathbf{0}$ are identity and zeros matrices, respectively, the dimensions of which are denoted by the corresponding subscripts.

Using exact discretization, the discrete-time state-space model is derived based on (5). This yields

$$
\begin{aligned}
\boldsymbol{x}(k+1) & =\boldsymbol{A} \boldsymbol{x}(k)+\boldsymbol{B} \boldsymbol{K} \boldsymbol{u}_{a b c}(k) \\
\boldsymbol{y}(k) & =\boldsymbol{C} \boldsymbol{x}(k),
\end{aligned}
$$

with $\boldsymbol{A}=\mathrm{e}^{\boldsymbol{F} T_{s}}$ and $\boldsymbol{B}=-\boldsymbol{F}^{-1}\left(\boldsymbol{I}_{8}-\boldsymbol{A}\right) \boldsymbol{G}$, where $\mathrm{e}$ is the matrix exponential, $T_{s}$ the sampling interval, and $k \in \mathbb{N}$.

\section{Direct MPC With FiXed Switching Frequency FOR CONTINUOUS MODUlation}

The proposed MPC strategy is a direct control method, thus the converter switches are directly manipulated. In the sequel, however, it is shown that despite the lack of a modulation stage, a fixed switching frequency can be achieved. 


\section{A. Control Problem}

The control objective of the controller is twofold. First, the controlled (i.e., output) variables need to be regulated along their reference values with as little deviation as possible. Second, the controller has to operate the converter at a constant switching frequency.

The first objective can be interpreted as minimizing the ripple of the output variables. This means that the weighted (squared) rms output error can be taken into account. Therefore, the objective function that captures this can be of the form ${ }^{1}$

$$
\begin{aligned}
J & =\frac{1}{T_{s}}\left(\int_{0}^{T_{s}}\left(\boldsymbol{y}_{\text {ref }}(t)-\boldsymbol{y}(t)\right)^{T} \boldsymbol{Q}\left(\boldsymbol{y}_{\text {ref }}(t)-\boldsymbol{y}(t)\right) \mathrm{d} t\right) \\
& =\frac{1}{T_{s}}\left(\int_{0}^{T_{s}}\left\|\boldsymbol{y}_{\text {ref }}(t)-\boldsymbol{y}(t)\right\|_{\boldsymbol{Q}}^{2} \mathrm{~d} t\right),
\end{aligned}
$$

with $\boldsymbol{y}_{\text {ref }}$ being the output reference vector, i.e., $\boldsymbol{y}_{\text {ref }}=\left[i_{\text {conv,ref }}^{T} \boldsymbol{i}_{g, \text { ref }}^{T} \boldsymbol{v}_{c, \text { ref }}^{T}\right]^{T} \in \mathbb{R}^{6}$, and $\boldsymbol{Q} \succ 0 \in \mathbb{R}^{6 \times 6}$ is a diagonal positive definite matrix, the entries of which prioritize the tracking accuracy among the different controlled variables. Note that, according to Parseval's theorem, minimization of (8) implies minimization of the (squared) total demand distortion (TDD) of the controlled variables $y$ over one sampling interval $T_{s}$.

To meet the second objective, thus ensuring a fixed switching frequency, each phase is forced to switch once per sampling interval $T_{s}$. To this end, we introduce the switching time instants $t_{1}, t_{2}$ and $t_{3}$, within a sampling interval such that $0<t_{1}<t_{2}<t_{3}<T_{s}$. At each of these time instants, a new switch position is applied to the converter. Specifically, let $\boldsymbol{u}_{a b c}\left(t_{0}\right)$ being the three-phase switch position applied at $t_{0} \equiv 0$. This switch position is assumed to be the same as the one applied to the converter at the end of the previous sampling interval, i.e., $\boldsymbol{u}_{a b c}\left(t_{0}\right)=\boldsymbol{u}_{a b c}\left(t_{0}^{-}\right)$. At time instant $t_{1}$, the switch position $\boldsymbol{u}_{a b c}\left(t_{1}\right)$ is applied which results in one commutation in one of the three phases. The switch position changes to $\boldsymbol{u}_{a b c}\left(t_{2}\right)$ at $t_{2}$ to alter the state in one of the two yet inactive phases. Finally, at $t_{3}$, the switch position $\boldsymbol{u}_{a b c}\left(t_{3}\right)$ is applied which forces the third-thus far inactive-phase to switch. To better understand this, the following example is given.

Example 1. Consider the grid-connected system in Fig. 1. Assume that the switch position applied at the end of the previous sampling interval was $\boldsymbol{u}_{a b c}\left(t_{0}^{-}\right)=[-1-1-1]^{T}$, as shown in Fig. 3(a). This means that at the beginning of the current sampling interval $\left(t_{0} \equiv 0\right)$, the same switch position, i.e., $\boldsymbol{u}_{a b c}\left(t_{0}\right)=\boldsymbol{u}_{a b c}\left(t_{0}^{-}\right)$, is applied. Within the sampling interval the switch positions $\boldsymbol{u}_{a b c}\left(t_{1}\right)=[1-1-1]^{T}$, $\boldsymbol{u}_{a b c}\left(t_{2}\right)=\left[\begin{array}{lll}1 & -1 & 1\end{array}\right]^{T}$ and $\boldsymbol{u}_{a b c}\left(t_{3}\right)=\left[\begin{array}{lll}1 & 1 & 1\end{array}\right]^{T}$ are applied at time instants $t_{1}, t_{2}$, and $t_{3}$, with $0<t_{1}<t_{2}<t_{3}<T_{s}$, respectively, see Fig. 3(a). As a result, the phases $a, c$, and $b$ switch (once) consecutively within $T_{s}$.

\footnotetext{
${ }^{1}$ The squared norm weighted with the positive (semi)definite matrix $\boldsymbol{W}$ is given by $\|\boldsymbol{\xi}\|_{\boldsymbol{W}}^{2}=\boldsymbol{\xi}^{T} \boldsymbol{W} \boldsymbol{\xi}$.
}

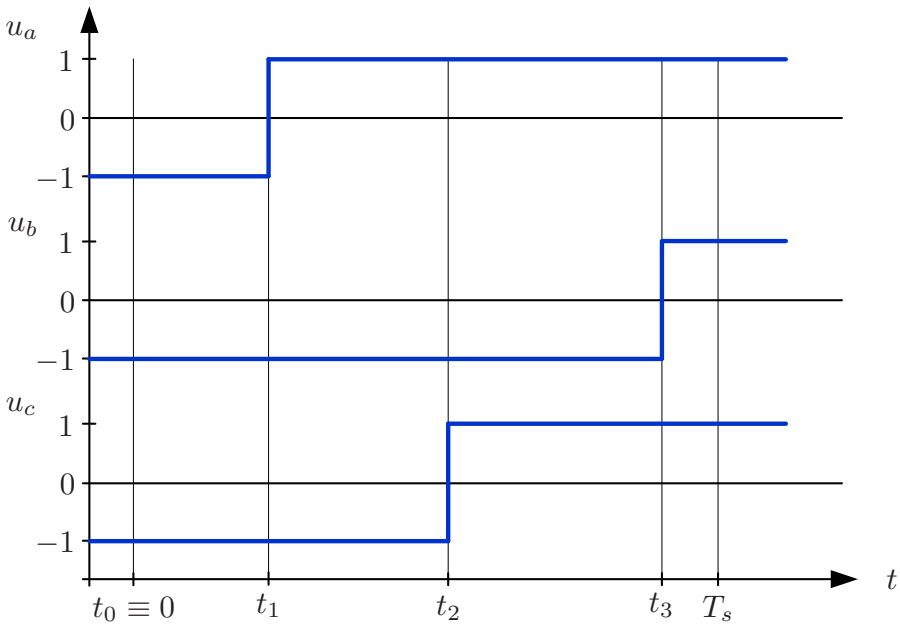

(a) Three-phase switch position

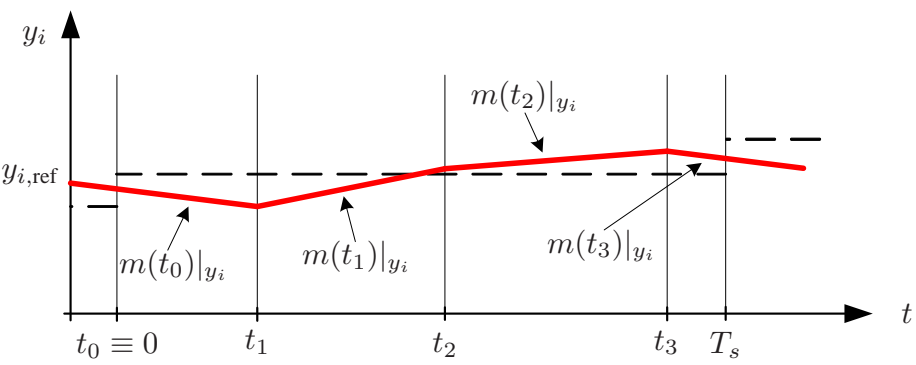

(b) Output variable $y_{i}$

Fig. 3: Example of the evolution of an (arbitrary) controlled variable $y_{i}$ within one $T_{s}$ by applying the depicted switching sequence $\boldsymbol{U}$, assuming $\boldsymbol{u}_{a b c}\left(t_{0}\right)=$ $\boldsymbol{u}_{a b c}\left(t_{0}^{-}\right)=[-1-1-1]^{T}$ and the phase sequence $a \rightarrow c \rightarrow b$.

Based on the above, the vector of switch positions $U$, i.e., the switching sequence, and the vector of switching time instants are defined as

$$
\begin{aligned}
\boldsymbol{U} & =\left[\begin{array}{llll}
\boldsymbol{u}_{a b c}^{T}\left(t_{0}\right) & \boldsymbol{u}_{a b c}^{T}\left(t_{1}\right) & \boldsymbol{u}_{a b c}^{T}\left(t_{2}\right) & \boldsymbol{u}_{a b c}^{T}\left(t_{3}\right)
\end{array}\right]^{T}, \\
\boldsymbol{t} & =\left[\begin{array}{lll}
t_{1} & t_{2} & t_{3}
\end{array}\right]^{T} .
\end{aligned}
$$

The three switching instants divide the switching interval $\left[0, T_{s}\right)$ into four subintervals $\left[0, t_{1}\right),\left[t_{1}, t_{2}\right),\left[t_{2}, t_{3}\right)$ and $\left[t_{3}, T_{s}\right)$. The three phases can switch in six different chronological orders, e.g., assuming phase $a$ switches first, either phase $b$ has to switch next, followed by phase $c$, or vice versa. Considering the different combinations with which all three phases can switch within on $T_{s}$, we conclude that six possible switching sequences $\boldsymbol{U}$ exist for a given $\boldsymbol{u}_{a b c}\left(t_{0}\right)$.

\section{B. Control Method}

In a next step, function (8) needs to be minimized for the six admissible switching sequences $\boldsymbol{U}$ as defined in (9a) to yield the corresponding vectors of switching time instants $t$ (9b). To do so, the evolution of the output $\boldsymbol{y}$ within the four subintervals needs to be computed for each $\boldsymbol{U}$. To simplify this task, and given that $T_{s} \ll T_{1}$, where $T_{1}$ is the fundamental period, the assumption that the output variables evolve linearly within $T_{s}$ is made. Hence, the evolution of the output variables within 
the four subintervals can be described by their corresponding (constant) gradients $\boldsymbol{m}(t)=\frac{\mathrm{d} \boldsymbol{y}(t)}{\mathrm{d} t} \in \mathbb{R}^{6}$, i.e.,

$\boldsymbol{m}\left(t_{i}\right)=\frac{\mathrm{d} \boldsymbol{y}\left(t_{i}\right)}{\mathrm{d} t}=\boldsymbol{C} \frac{\mathrm{d} \boldsymbol{x}\left(t_{i}\right)}{\mathrm{d} t}=\boldsymbol{C}\left(\boldsymbol{F} \boldsymbol{x}\left(t_{0}\right)+\boldsymbol{G} \boldsymbol{K} \boldsymbol{u}_{a b c}\left(t_{i}\right)\right)$,

where $i \in\{0,1,2,3\}$. It should be noted that in (10), the gradients at $t_{1}, t_{2}$ and $t_{3}$ depend on the state at time instant $t_{0}$, i.e., $\boldsymbol{x}\left(t_{0}\right)$, (rather than on $\boldsymbol{x}\left(t_{1}\right), \boldsymbol{x}\left(t_{2}\right)$, and $\boldsymbol{x}\left(t_{3}\right)$, respectively) because of the aforementioned assumption of constant gradients within the sampling interval.

Example 2. Consider the switching sequence $\boldsymbol{U}$ depicted in Fig. 3(a). By applying this sequence, the evolution of the controlled variables changes, and it can thus be controlled. Assuming piecewise affine output trajectories-and thus a piecewise constant gradient $\boldsymbol{m}(t)$-the continuoustime evolution of one (arbitrary) controlled variable $y_{i}$, with $i \in\{1,2, \ldots, 6\}$, is shown, along with its corresponding reference, in Fig. 3(b).

With (10), the objective function (8) is simplified. However, the problem is nonconvex since (8) is a cubic function of time. To turn the problem into a convex one, we choose to further simplify (8) by penalizing - instead of the (weighted) rms error-the deviation of the controlled variables from their references at the switching instants and at the end of sampling interval, see [33]. By doing so, the rms output error is approximated in a coarse yet effective manner. Moreover, by considering the error only at the switching instants and discrete time steps, the computational complexity of the problem is kept modest.

In light of the above approximation, function (8) becomes

$$
J=\sum_{i=1}^{3}\left\|\boldsymbol{y}_{\mathrm{ref}}\left(t_{i}\right)-\boldsymbol{y}\left(t_{i}\right)\right\|_{\boldsymbol{Q}}^{2}+\left\|\boldsymbol{y}_{\mathrm{ref}}\left(T_{s}\right)-\boldsymbol{y}\left(T_{s}\right)\right\|_{\boldsymbol{Q}}^{2},
$$

However, (11) is further modified to improve the tracking performance of the controller. Specifically, the aim of the following refinements is to eliminate undesired harmonics that violate the relevant grid codes, such as the IEEE 519 standard [35].

1) Longer Prediction Horizon: In [38], it is shown that longer prediction horizons lead to better system performance by predicting the system behavior further into the future. This benefit becomes even more evident when considering higher order MIMO systems, such as the examined case study.

In this work, a two-step prediction horizon is implemented. Accordingly, the switching sequence and vector of switching time instants are redefined as

$$
\begin{aligned}
\boldsymbol{U} & =\left[\begin{array}{ll}
\boldsymbol{U}^{T}(k) & \boldsymbol{U}^{T}(k+1)
\end{array}\right]^{T}, \\
\boldsymbol{t} & =\left[\begin{array}{ll}
\boldsymbol{t}^{T}(k) & \boldsymbol{t}^{T}(k+1)
\end{array}\right]^{T} .
\end{aligned}
$$

where

$\boldsymbol{U}(\ell)=\left[\boldsymbol{u}_{a b c}^{T}\left(t_{0}(\ell)\right) \boldsymbol{u}_{a b c}^{T}\left(t_{1}(\ell)\right) \boldsymbol{u}_{a b c}^{T}\left(t_{2}(\ell)\right) \boldsymbol{u}_{a b c}^{T}\left(t_{3}(\ell)\right)\right]^{T}$, and

$$
\boldsymbol{t}(\ell)=\left[\begin{array}{lll}
t_{1}(\ell) & t_{2}(\ell) & t_{3}(\ell)
\end{array}\right]^{T}
$$

\begin{tabular}{|c|c|c|c|c|c|}
\hline \multicolumn{6}{|c|}{ Phases to switch } \\
\hline \multicolumn{3}{|c|}{$1^{\text {st }}$ sampling interval } & \multicolumn{3}{|c|}{$2^{\text {nd }}$ sampling interval } \\
\hline First & Second & Third & First & Second & Third \\
\hline$a$ & $b$ & $c$ & $c$ & $b$ & $a$ \\
\hline$a$ & $c$ & $b$ & $b$ & $c$ & $a$ \\
\hline$b$ & $a$ & $c$ & $c$ & $a$ & $b$ \\
\hline$b$ & $c$ & $a$ & $a$ & $c$ & $b$ \\
\hline$c$ & $a$ & $b$ & $b$ & $a$ & $c$ \\
\hline$c$ & $b$ & $a$ & $a$ & $b$ & $c$ \\
\hline
\end{tabular}

TABLE I: Possible sequences for the single-phase switch transitions for a two-step horizon

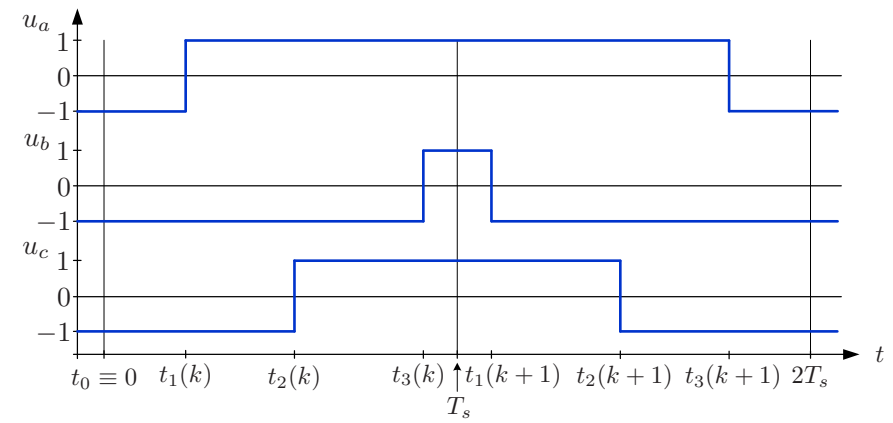

Fig. 4: Example of a prediction horizon over two sampling intervals with six switching instants.

with $\ell=k, k+1$. As implied by (12a), the number of possible switching sequences is squared, i.e., $6^{2}=36$ switching sequences should be considered for a two-step prediction horizon. To keep the computational complexity at bay, we assume that the switching sequence in the second sampling interval $\boldsymbol{U}(k+1)$ mirrors that of the first sampling interval $\boldsymbol{U}(k)$ with respect to $T_{s}$, akin, e.g., to the SVM switching pattern. This means that $\boldsymbol{U}(k+1)=$ $\left[\boldsymbol{u}_{a b c}^{T}\left(t_{3}(k)\right) \boldsymbol{u}_{a b c}^{T}\left(t_{2}(k)\right) \boldsymbol{u}_{a b c}^{T}\left(t_{1}(k)\right) \boldsymbol{u}_{a b c}^{T}\left(t_{0}(k)\right)\right]^{T}$, as depicted in Fig. 4. Consequently, the number of possible switching sequences remains equal to six. The possible switching sequences for the single-phase switch transitions over a twostep prediction horizon are given in Table I.

2) Linear Approximation of the References: Since the references are sinusoidally varying quantities, the sample-and-hold approach, i.e., the assumption of constant references at each sampling interval, similar to SVM or asymmetric regularly sampled CB-PWM, would result in tracking errors. A better approximation is to linearly interpolate the references between two consecutive discrete time steps, i.e., between $k$ and $k+1$ as well as between $k+1$ and $k+2$. This yields

$$
\boldsymbol{y}_{\mathrm{ref}}(t)=\boldsymbol{y}_{\mathrm{ref}}(\ell)+\boldsymbol{m}_{\mathrm{ref}}(\ell) t
$$

where

$$
\boldsymbol{m}_{\mathrm{ref}}(\ell)=\frac{\boldsymbol{y}_{\mathrm{ref}}(\ell+1)-\boldsymbol{y}_{\mathrm{ref}}(\ell)}{T_{s}}
$$

and $\ell=k, k+1$. This process is visualized in Fig. 5 .

3) Heavier Penalization of the Discrete Time Steps: For the sake of simplicity — but without loss of generality - a gridconnected converter with an $L$ filter serves as an example for 


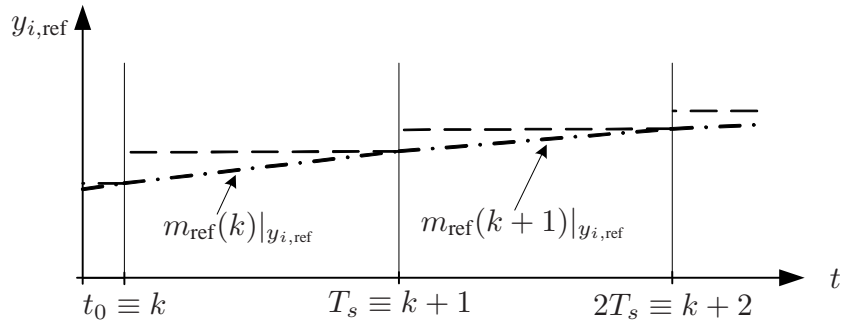

Fig. 5: Linear approximation of the reference value of a (arbitrary) controlled variable $y_{i, \text { ref }}$ over two sampling intervals (dash-dotted line). The sampled (constant) reference is shown with a dashed line.

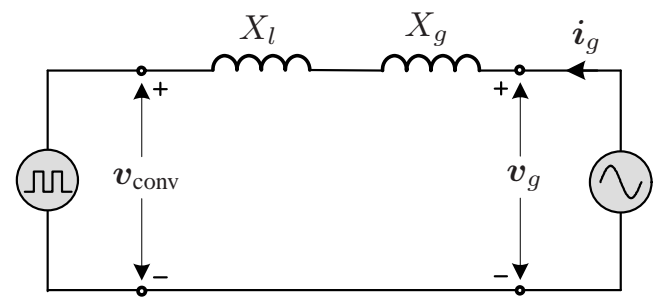

Fig. 6: Equivalent circuit of the grid-connected converter with an $L$ filter in the stationary $(\alpha \beta)$ frame.

analyzing this concept. Considering the equivalent circuit of the system, ${ }^{2}$ as shown in Fig. 6, the differential equation

$$
\frac{\mathrm{d} \boldsymbol{i}_{g}}{\mathrm{~d} t}=\frac{1}{X_{l}+X_{g}}\left(\boldsymbol{v}_{g}-\boldsymbol{v}_{\text {conv }}\right) \text {. }
$$

describes the dynamics of the grid current, where $X_{l}$ denotes the filter reactance.

The grid voltage in the stationary $(\alpha \beta)$ reference frame is a vector which rotates counterclockwise with the angular speed $\omega_{g}=2 \pi f_{1}$, with $f_{1}$ being the fundamental frequency. Focusing on one instant of the problem, the following assumptions are made to enhance the clarity of the analysis that follows:

- The grid voltage $\boldsymbol{v}_{g}$ is located in the first sector (i.e., the triangle formed by $\boldsymbol{v}_{1}, \boldsymbol{v}_{2}$, and $\boldsymbol{v}_{0,7}$ ) of the hexagon formed by the voltage vectors $\boldsymbol{v}_{\text {conv }}$ that can be produced by the converter (2), see Fig. 7(a).

- As implied by Figs. 3(a) and 4, at the beginning of the sampling interval either the switch position $\boldsymbol{u}_{a b c}\left(t_{0}\right)=[-1-1-1]^{T}$ (i.e., voltage vector $\boldsymbol{v}_{0}$ ), or $\boldsymbol{u}_{a b c}=\left[\begin{array}{lll}1 & 1 & 1\end{array}\right]^{T}$ (i.e., voltage vector $\boldsymbol{v}_{7}$ ) is applied to the converter. Assume that $\boldsymbol{v}_{0}$ is applied at $t_{0}$.

- The phase sequence $a \rightarrow b \rightarrow c$ is implemented. Therefore, the consecutively applied switch positions within $T_{s}, \boldsymbol{u}_{a b c}\left(t_{1}\right), \boldsymbol{u}_{a b c}\left(t_{2}\right)$, and $\boldsymbol{u}_{a b c}\left(t_{3}\right)$, correspond to the voltage vectors $\boldsymbol{v}_{1}, \boldsymbol{v}_{2}$, and $\boldsymbol{v}_{7}$, respectively.

- The grid current ripple at $t_{0}$ is zero, i.e., $\boldsymbol{i}_{g, \text { rip }}\left(t_{0}\right)=\mathbf{0}$, where $i_{g \text {,rip }}=i_{g}-i_{g 1}$, with $i_{g 1}$ being the fundamental component of $\boldsymbol{i}_{g}$.

Based on the above, if the trajectories of the grid current $\mathrm{d} \boldsymbol{i}_{g}\left(\boldsymbol{v}_{\text {conv }}\right) / \mathrm{d} t$ are approximated as linear, then the trajectory of the grid current ripple $\boldsymbol{i}_{g, \text { rip }}$ ideally will be as the one shown in Fig. 7(b). Such a trajectory-which can be produced with,

\footnotetext{
${ }^{2}$ Note that due to their small values, the filter and grid resistances are neglected in the analysis presented.

${ }^{3}$ Dropping these assumptions so as to generalize the analysis is straightforward.
}

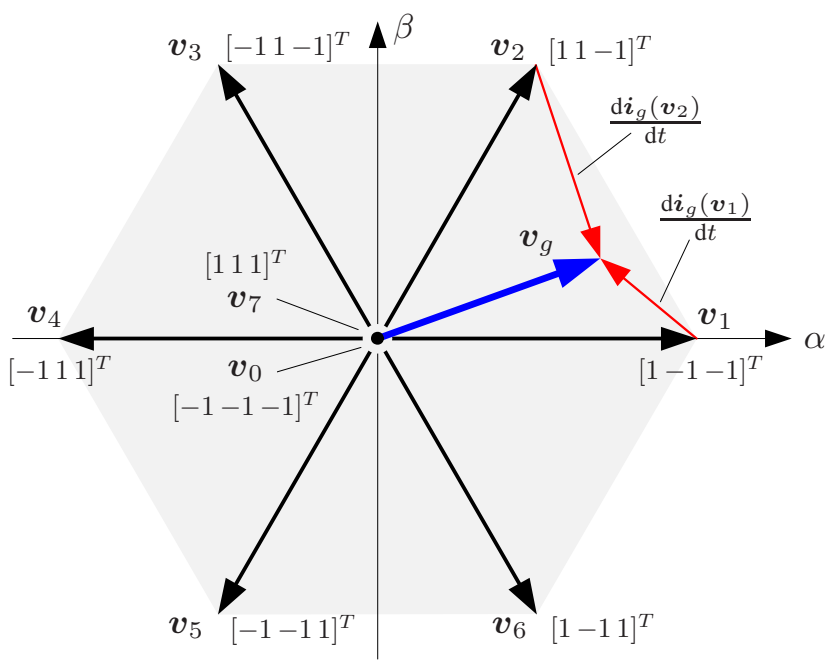

(a) Two-level converter and grid voltage vectors in stationary reference frame $(\alpha \beta)$

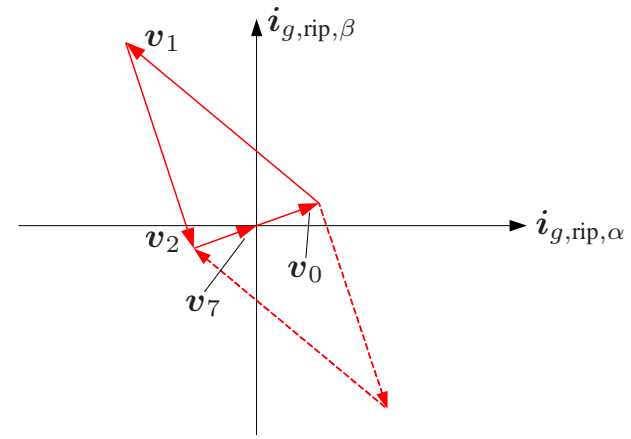

(b) Ideal ripple of the current.

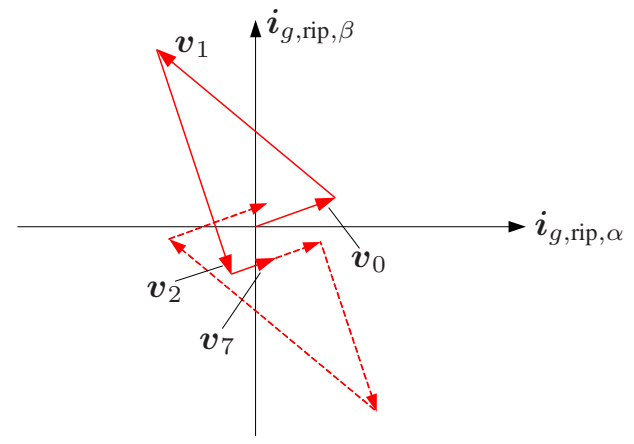

(c) Actual ripple of the current when the discrete time steps are not penalized more heavily.

Fig. 7: Linearized trajectory of the grid current ripple over two consecutive sampling intervals $T_{s}$ assuming the depicted vector of the voltage grid $\boldsymbol{v}_{g}$. The trajectories over the first and second $T_{s}$ (modulation half-cycles) are shown with solid and dashed lines, respectively.

e.g., SVM-indicates that the current error (i.e., ripple) is zero at the discrete time steps $k, k+1, k+2, \ldots$. With the proposed MPC, nonetheless, a zero ripple at the discrete time steps is not guaranteed since the rms of the output error is chosen to be minimized instead. Consequently, a current ripple trajectory as the one depicted in Fig. 7(c) is likely to result, which implies that undesired low frequency harmonics appear due to the fact that symmetry in the implemented switching sequences is not enforced.

To overcome the aforementioned issue, and to thus ensure 


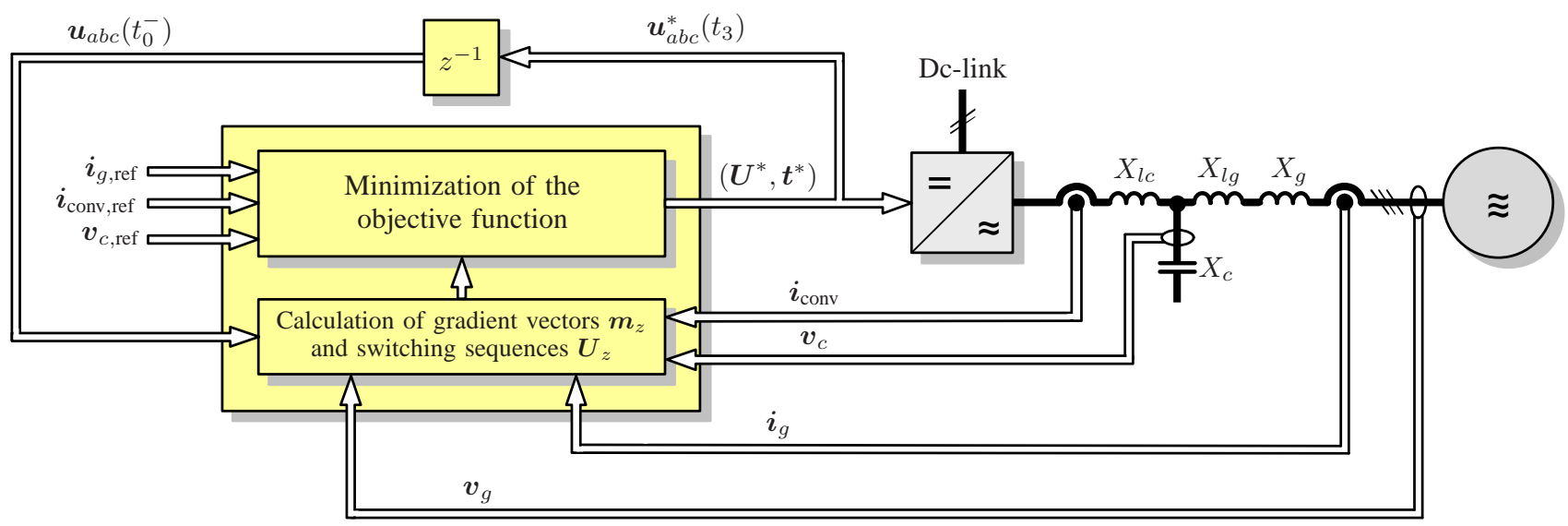

Fig. 8: Direct model predictive control with fixed switching frequency for a two-level grid-connected converter with an $L C L$ filter.

that the grid current ripple trajectory returns to the origin of the plane at the end of each sampling interval (see Fig. 7(b)), it is proposed to heavily penalize the output error at the discrete time steps. Therefore, when applying this approach to the examined case study, i.e., grid-connected converter with an $L C L$ filter, see Fig. 2, the deviation of all controlled variables $\boldsymbol{y}$ from their reference values is more heavily penalized at the discrete time steps.

\section{Objective Function}

Taking into account all the assumptions and refinements presented in Section III-B, the objective function is defined as

$$
\begin{aligned}
J=\sum_{\ell=k}^{k+1} & \left(\sum_{i=1}^{3}\left\|\boldsymbol{y}_{\mathrm{ref}}\left(t_{i}(\ell)\right)-\boldsymbol{y}\left(t_{i}(\ell)\right)\right\|_{\boldsymbol{Q}}^{2}+\right. \\
& \left.+\left\|\boldsymbol{\Lambda}\left(\boldsymbol{y}_{\mathrm{ref}}\left(T_{s}(\ell)\right)-\boldsymbol{y}\left(T_{s}(\ell)\right)\right)\right\|_{\boldsymbol{Q}}^{2}\right),
\end{aligned}
$$

where the references values $\boldsymbol{y}_{\text {ref }}$ are computed based on (13). Moreover, $\boldsymbol{\Lambda} \succ 0 \in \mathbb{R}^{6 \times 6}$ is a diagonal positive definite matrix the entries of which assign higher priority to the tracking accuracy at the discrete time steps. Therefore, its nonzero entries are greater than one.

Utilizing (10), it is straightforward to show that the output variables can be written as

$$
\boldsymbol{y}\left(t_{i}(\ell)\right)=\boldsymbol{y}\left(t_{i-1}(\ell)\right)+\boldsymbol{m}\left(t_{i-1}(\ell)\right)\left(t_{i}(\ell)-t_{i-1}(\ell)\right)
$$

with $\ell=k, k+1, i \in\{1,2,3,4\}$ and $t_{4}=T_{s}$. As shown in [33], (16), after some algebraic manipulations, and with the aid of (17), can be written in vector form as

$$
J=\|\boldsymbol{r}-\boldsymbol{M t}\|_{\widetilde{\boldsymbol{Q}}}^{2},
$$

where the vector of switching instants $t \in \mathbb{R}^{6}$, as given by (12b), is the optimization (unknown) variable. Furthermore, vector $\boldsymbol{r} \in \mathbb{R}^{48}$ and matrix $M$ are time invariant and given in the appendix. Finally, $\widetilde{\boldsymbol{Q}}=\operatorname{diag}(\boldsymbol{Q}, \ldots, \boldsymbol{Q})$. $\overline{\text { Algorithm } 1 \text { Direct MPC with fixed switching frequency and }}$ continuous modulation

Given $\boldsymbol{u}_{a b c}\left(t_{0}^{-}\right), \boldsymbol{y}_{\text {ref }}\left(t_{0}\right)$ and $\boldsymbol{x}\left(t_{0}\right)$

0. Compute the corresponding gradient vectors $\boldsymbol{m}_{z}, z \in\{0,1, \ldots, 6\}$.

1. Enumerate the possible switching sequences $\boldsymbol{U}_{z}, z \in\{1,2, \ldots, 6\}$, starting from $\boldsymbol{u}_{a b c}\left(t_{0}^{-}\right)$.

2. For each $\boldsymbol{U}_{z}$ :

Solve the QP (20). This yields $\boldsymbol{t}_{z}$ and $J_{z}$. 3. Solve the trivial optimization problem (21). This yields $\boldsymbol{t}^{*}$ and $\boldsymbol{U}^{*}$. Return $\boldsymbol{U}^{*}(k)$ and $\boldsymbol{t}^{*}(k)$

\section{Control Algorithm}

The block diagram of the proposed direct MPC scheme is shown in Fig. 8. Moreover, the pseudocode of the control method is summarized in Algorithm 1. In the sequel of this section, the algorithm is explained in detail.

Before the control algorithm is executed, the possible gradients that depend on the measured state vector $\boldsymbol{x}\left(t_{0}\right)$ and the possible switch positions $\boldsymbol{u}_{a b c}$ of the two-level converter are computed. Hence, seven unique output vector gradients $\boldsymbol{m}_{z}$, with $z \in\{0,1, \ldots, 6\}$, need to be computed, since the eight possible switch positions $\boldsymbol{u}_{a b c}$ of the converter yield seven different voltage vectors in the $\alpha \beta$-plane, see Fig. 7(a). To this end, (10) is written as

$$
\boldsymbol{m}_{z}=\boldsymbol{C}\left(\boldsymbol{F} \boldsymbol{x}\left(t_{0}\right)+\boldsymbol{G} \boldsymbol{u}_{z}\right),
$$

where $\boldsymbol{u}_{z}$ refers to the seven different switch positions in the $\alpha \beta$-plane, i.e., $\boldsymbol{u}_{z}=\boldsymbol{K} \boldsymbol{u}_{a b c, z}$.

Having computed the output vector gradients (19), the controller enumerates the possible three-phase switch positions within the two sampling intervals based on the previously applied switch position $\boldsymbol{u}_{a b c}\left(t_{0}^{-}\right)$and in line with Table I. This yields the six feasible switching sequences $\boldsymbol{U}_{z}, z \in$ $\{1,2, \ldots, 6\}$.

In a second step, the optimization problem

$$
\begin{aligned}
\underset{\boldsymbol{t} \in \mathbb{R}^{6}}{\operatorname{minimize}} & \|\boldsymbol{r}-\boldsymbol{M} \boldsymbol{t}\|_{\widetilde{\boldsymbol{Q}}}^{2} \\
\text { subject to } & 0<t_{1}(k)<t_{2}(k)<t_{3}(k)<T_{s}< \\
& <t_{1}(k+1)<t_{2}(k+1)<t_{3}(k+1)<2 T_{s} .
\end{aligned}
$$


is solved for each one of the six sequences $\boldsymbol{U}_{z}$. Problem (20) is a convex quadratic program (QP) [39]. Owing to its small size (the optimization variable is six-dimensional), it can be solved efficiently using online QP solvers that can solve such MPC problems on embedded hardware even in a manner of microseconds, see, e.g., [40]-[43]. The solution of each QP is a triplet of switching instants $\boldsymbol{t}_{z}(12 \mathrm{~b})$, switching sequence $U_{z}(12 \mathrm{a})$, and the associated value of the objective function $J_{z}$ (18), with $z \in\{1,2, \ldots, 6\}$.

In a last step, the algorithm decides which one of the six triplets $\left\{\boldsymbol{t}_{z}, \boldsymbol{U}_{z}, J_{z}\right\}$ has the minimal value of the objective function. This is done by solving the following trivial optimization problem

$$
\underset{z \in\{1,2, \ldots, 6\}}{\operatorname{minimize}} J_{z}
$$

By doing so, the triplet that meets $J\left(U^{*}, t^{*}\right)=J^{*}$ is the optimal one, i.e., $\left\{\boldsymbol{t}^{*}, \boldsymbol{U}^{*}, J^{*}\right\}$. Following, and according to the receding horizon policy [1], only the elements corresponding to the first sampling interval, i.e., $\boldsymbol{U}^{*}(k)$ and $\boldsymbol{t}^{*}(k)$, are taken into account, while the rest are discarded. Thus, the first part of the optimal switching sequence is applied with the appropriate switching times to the converter, i.e.,

$$
\begin{aligned}
\boldsymbol{t}^{*}(k) & =\left[\begin{array}{lll}
t_{1}^{*}(k) & t_{2}^{*}(k) & t_{3}^{*}(k)
\end{array}\right]^{T} \\
\boldsymbol{U}^{*}(k) & =\left[\begin{array}{ll}
\boldsymbol{u}_{a b c}^{* T}\left(t_{0}(k)\right) \boldsymbol{u}_{a b c}^{* T}\left(t_{1}^{*}(k)\right) \boldsymbol{u}_{a b c}^{* T}\left(t_{2}^{*}(k)\right) \boldsymbol{u}_{a b c}^{* T}\left(t_{3}^{*}(k)\right)
\end{array}\right]^{T} .
\end{aligned}
$$

At the next sampling interval, the algorithm is repeated based on new measurements over a prediction horizon shifted by one sampling interval.

\section{DIRECT MPC WITH FIXED SWITCHING FREQUENCY FOR DISCONTINUOUS MODULATION}

To reduce the converter switching losses and, consequently, to improve the system efficiency, the switching frequency needs to be kept low. To meet this goal, DPWM can be employed to avoid switching in the vicinity of phase current peaks [44]. The most straightforward approach to achieve this is to force the three phases to consecutively refrain from switching for one-third of the fundamental period, i.e., to keep each phase leg clamped to the negative dc rail for $120^{\circ}$ of the fundamental cycle. As a result, with DPWMMIN, as the method is called, the switching frequency-and thus the switching losses-are reduced by $33 \%$ compared with continuous modulation techniques that use the same carrier frequency. Nonetheless, this comes at the cost of unequal distribution of the power losses between the upper and the lower switches of the phase legs [34].

Motivated by the aforementioned attributes of discontinuous modulation, the direct MPC strategy discussed in Section III is modified in this section to emulate the switching pattern of DPWMMIN.

\section{A. Control Problem}

Besides the control objectives defined in Section III-A, i.e., the minimization of the rms error of the controlled variables $\boldsymbol{y}$ as well as the converter operation at a fixed switching frequency, the additional task of the reduced switching frequency is considered. The ultimate goal of the proposed MPC algorithm is to operate the converter at the lowest possible switching frequency while meeting the relevant grid codes [35].

Considering the above, the control problem has similarities with that discussed in Section III-A. Therefore, the control principle and introduced concepts, such as the switching sequence $\boldsymbol{U}$ and instants $\boldsymbol{t}$, remain in place. For MPC, however, to emulate the switching pattern of DPWMMIN, one of the three phases has to remain inactive, i.e., clamped to the negative dc rail, for the whole sampling interval. This implies that only two different switch positions should be applied to the converter at two consecutive switching instants within one $T_{s}$. Consequently, for direct MPC with discontinuous modulation, the switching sequence $\boldsymbol{U}(\ell)$ and vector of switching instants $\boldsymbol{t}(\ell)$ in (12a) and (12b), respectively, are defined as

$$
\begin{aligned}
\boldsymbol{U}(\ell) & =\left[\begin{array}{lll}
\boldsymbol{u}_{a b c}^{T}\left(t_{0}(\ell)\right) & \boldsymbol{u}_{a b c}^{T}\left(t_{1}(\ell)\right) & \boldsymbol{u}_{a b c}^{T}\left(t_{2}(\ell)\right)
\end{array}\right]^{T} \\
\boldsymbol{t}(\ell) & =\left[\begin{array}{ll}
t_{1}(\ell) & t_{2}(\ell)
\end{array}\right]^{T} .
\end{aligned}
$$

By doing so, it is ensured that only two phases switch within one $T_{s}$. This, however, does not suffice to guarantee that one phase remains inactive for one third of the fundamental period, as it is the case with DPWMMIN. To address this, further modifications are required, as explained in Section IV-B.

\section{B. Control Method}

DPWMMIN utilizes only one of the two zero voltage vectors of a two-level converter (Fig. 9), i.e., the voltage vector $\boldsymbol{v}_{0}$. Assume that the proposed MPC algorithm implements the corresponding switch position at the beginning of the sampling interval, i.e., $\boldsymbol{u}_{a b c}\left(t_{0}(k)\right)=\left[\begin{array}{lll}-1 & -1-1\end{array}\right]^{T}$. Then, after applying two switch positions that activate as many phases at instants $t_{1}(k)$ and $t_{2}(k)$, the single-phase switch position of the third (inactive) phase will still be -1 by the end of the interval, $u_{x}\left(T_{s}\right)=-1$.

As can be understood from the above, the proposed algorithm has not only to compute the appropriate switching time instants and sequence, but also to identify which phase should be kept inactive within the sampling interval. To achieve the latter, a deadbeat approach is employed. More specifically, the ideal converter output voltage $\boldsymbol{v}_{\text {conv,ref }}(k)$ that drives the converter current to its reference within one sampling interval is computed. Using forward Euler discretization, (4a) can be written as

$$
\begin{aligned}
\frac{\boldsymbol{i}_{\text {conv }}(k+1)-\boldsymbol{i}_{\text {conv }}(k)}{T_{s}} & =\frac{1}{X_{l c}}\left(\boldsymbol{v}_{c}(k)-\left(R_{l c}+R_{c}\right) \boldsymbol{i}_{\text {conv }}(k)+\right. \\
& \left.+R_{c} \boldsymbol{i}_{g}(k)-\boldsymbol{v}_{\text {conv }}(k)\right) .
\end{aligned}
$$

Setting as a goal $\boldsymbol{i}_{\text {conv }}(k+1)=\boldsymbol{i}_{\text {conv,ref }}(k+1), \boldsymbol{v}_{\text {conv,ref }}(k)$ is given by

$$
\begin{aligned}
\boldsymbol{v}_{\text {conv,ref }}(k) & =\boldsymbol{v}_{c}(k)+R_{c} \boldsymbol{i}_{g}(k)-\frac{X_{l c}}{T_{s}} \boldsymbol{i}_{\text {conv,ref }}(k+1)+ \\
& +\left(\frac{X_{l c}}{T_{s}}-\left(R_{l c}+R_{c}\right)\right) \boldsymbol{i}_{\mathrm{conv}}(k) .
\end{aligned}
$$




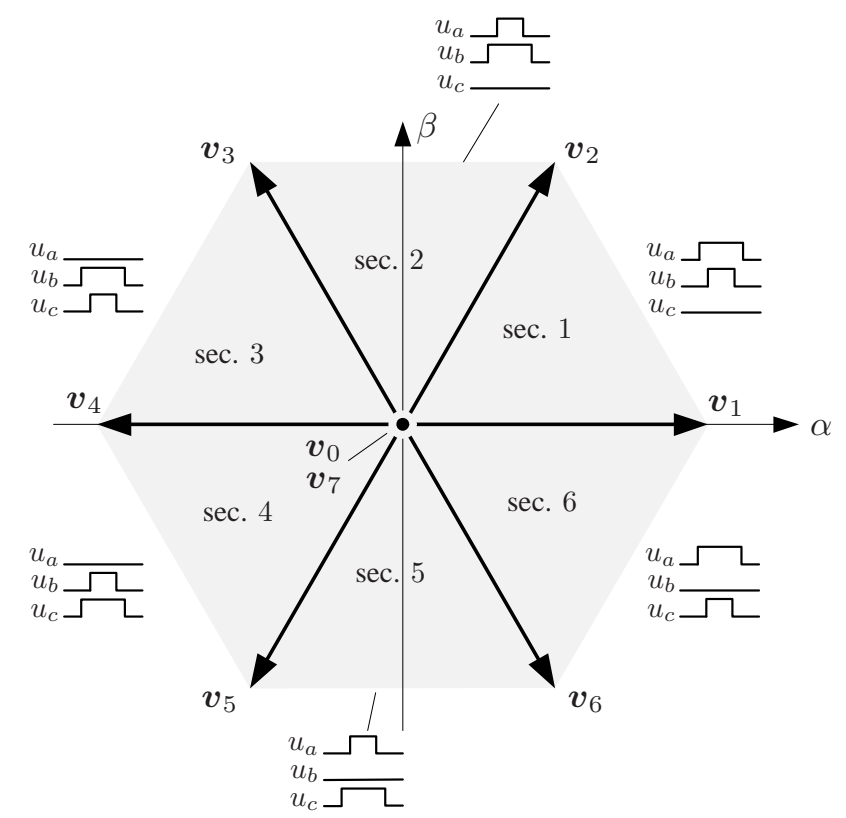

Fig. 9: Two-level converter voltage vector diagram and switching patterns per sector according to DPWMMIN.

Following, the inactive phase is determined based on the location of $\boldsymbol{v}_{\text {conv,ref }}(k)$ in the $\alpha \beta$-plane, i.e., the angle $\angle \boldsymbol{v}_{\text {conv,ref }}(k)$. Specifically, when $\boldsymbol{v}_{\text {conv,ref }}(k)$ is located at sectors 1 or 2, see Fig. 9, phase leg $c$ is clamped to the $-1\left(u_{c}=-1\right)$. Thereby, only phases $a$ and $b$ are allowed to switch. If, on the other hand, $\boldsymbol{v}_{\text {conv,ref }}(k)$ is at sectors 3 or 4 , then $u_{a}=-1$ and phases $b$ and $c$ are active. Finally, for the last two sectors, phases $a$ and $c$ switch, while $u_{b}=-1$.

Having determined the phase clamped to the negative dc rail, the possible switching sequences are generated. Therefore, by excluding the inactive phase, the two remaining phases can switch in two possible chronological orders. This means that at any given instant, only two-in contrast to six, which is the case with MPC with continuous modulation-switching sequences $\boldsymbol{U}$ need to be examined. If, e.g., $\boldsymbol{v}_{\text {conv,ref }}(k)$ is located at the first sector, the two active phases switch such that either phase $a$ switches first and $b$ follows, or vice versa. The active phases, depending on the location of $\boldsymbol{v}_{\text {conv,ref }}(k)$, as well as the possible sequences over one $T_{s}$ are summarized in the first three columns of Table II.

Besides the above-mentioned modifications, the refinements introduced in Section III-B are utilized with MPC with discontinuous modulation. This means that the switching sequences over a two-step horizon $\left(N_{p}=2\right)$ are again mirrored with respect to $T_{s}$; the resulting possible switching sequences are shown in Table II. As seen in the table, the total number of possible switching sequences remains the same as with MPC with continuous modulation. However, as explained above, only two are taken into account each time.
TABLE II: Possible sequences for the single-phase switch transitions for a two-step horizon when emulating the switching pattern of DPWMMIN

\begin{tabular}{|c|cccc|}
\hline \multirow{2}{*}{$\begin{array}{c}\text { Location of } \\
\boldsymbol{v}_{\text {conv,ref }}(k)\end{array}$} & \multicolumn{4}{|c|}{ Phases to switch } \\
\cline { 2 - 5 } & $1^{\text {st }}$ sampling interval & $2^{\text {nd }}$ sampling interval \\
\cline { 2 - 5 } & First & Second & First & Second \\
\hline \multirow{2}{*}{ Sectors 1 or 2} & $a$ & $b$ & $b$ & $a$ \\
& $b$ & $a$ & $a$ & $b$ \\
\hline \multirow{2}{*}{ Sectors 3 or 4} & $b$ & $c$ & $c$ & $b$ \\
& $c$ & $b$ & $b$ & $c$ \\
\hline \multirow{2}{*}{ Sectors 5 or 6} & $a$ & $c$ & $c$ & $a$ \\
& $c$ & $a$ & $a$ & $c$ \\
\hline
\end{tabular}

$\overline{\text { Algorithm } 2 \text { Direct MPC with fixed switching frequency and }}$ discontinuous modulation

Given $\boldsymbol{u}_{a b c}\left(t_{0}^{-}\right), \boldsymbol{y}_{\text {ref }}\left(t_{0}\right)$ and $\boldsymbol{x}\left(t_{0}\right)$

0 . Compute the corresponding gradient vectors $\boldsymbol{m}_{z}, z \in\{0,1, \ldots, 6\}$.

1a. Identify the sector of the desired converter output voltage vector $\boldsymbol{v}_{\text {conv,ref }}(k)$ based on $\angle \boldsymbol{v}_{\text {conv,ref }}(k)$.

1b. Enumerate the possible switching sequences $\boldsymbol{U}_{z}, z \in\{1,2\}$, starting from $\boldsymbol{u}_{a b c}\left(t_{0}^{-}\right)$.

2. For each $U_{z}$ :

Solve the QP (27). This yields $\boldsymbol{t}_{z}$ and $J_{z}$.

3. Solve the trivial optimization problem (21) for $z \in\{1,2\}$. This yields $\boldsymbol{t}^{*}$ and $\boldsymbol{U}^{*}$

Return $\boldsymbol{U}^{*}(k)$ and $\boldsymbol{t}^{*}(k)$

Given the above, the objective function is

$$
\begin{aligned}
J=\sum_{\ell=k}^{k+1} & \left(\sum_{i=1}^{2}\left\|\boldsymbol{y}_{\text {ref }}\left(t_{i}(\ell)\right)-\boldsymbol{y}\left(t_{i}(\ell)\right)\right\|_{\boldsymbol{Q}}^{2}+\right. \\
& \left.+\left\|\boldsymbol{\Lambda}\left(\boldsymbol{y}_{\text {ref }}\left(T_{s}(\ell)\right)-\boldsymbol{y}\left(T_{s}(\ell)\right)\right)\right\|_{\boldsymbol{Q}}^{2}\right) .
\end{aligned}
$$

Finally, function (26) can be written in the same vector form as in (18). However, the size of the optimization problem underlying MPC is smaller. This is due to the fact that the optimization variable, i.e., the switching instants $t(23 \mathrm{~b})$, is a four-dimensional vector. Moreover, $r \in \mathbb{R}^{36}$, and matrix $\boldsymbol{M}$ is of appropriate dimensions.

\section{Control Algorithm}

As in Section III-D, in a preprocessing step, the possible output gradients $\boldsymbol{m}_{z}$, with $z \in\{0,1, \ldots, 6\}$, are computed based on (19).

Subsequently, the location of the desired converter output voltage (25) is identified, and the two candidate switching sequences are determined according to Table II. Depending on $\boldsymbol{u}_{a b c}\left(t_{0}^{-}\right)$, the controller enumerates the two feasible switching sequences $\boldsymbol{U}_{z}$, with $z \in\{1,2\}$.

Following, for each one of the two given switching sequence the optimization problem

$$
\begin{array}{cl}
\underset{\boldsymbol{t} \in \mathbb{R}^{4}}{\operatorname{minimize}} & \|\boldsymbol{r}-\boldsymbol{M} \boldsymbol{t}\|_{\widetilde{\boldsymbol{Q}}}^{2} \\
\text { subject to } & 0<t_{1}(k)<t_{2}(k)<T_{s}< \\
& <t_{1}(k+1)<t_{2}(k+1)<2 T_{s} .
\end{array}
$$

is solved. As before (see Section III-D), the solution of the QP is a triplet $\left\{\boldsymbol{t}_{z}, \boldsymbol{U}_{z}, J_{z}\right\}$, with $\boldsymbol{t}_{z}$ and $\boldsymbol{U}_{z}$ given by (23). 
TABLE III: Rated values of the system

\begin{tabular}{|ccc|}
\hline Parameter & Symbol & SI Value \\
\hline Voltage & $V_{R}$ & $400 \mathrm{~V}$ \\
Current & $I_{R}$ & $18 \mathrm{~A}$ \\
Angular grid frequency & $\omega_{g R}$ & $2 \pi 50 \mathrm{rad} / \mathrm{s}$ \\
Short-circuit ratio & $k_{\mathrm{sc}}$ & 20 \\
Grid impedance ratio & $k_{\mathrm{XR}}=X / R$ & 7 \\
\hline
\end{tabular}

TABLE IV: System parameters

\begin{tabular}{|c|ccc|}
\hline Grid & Reactance & $X_{g}$ & 0.0490 \\
& Resistance & $R_{g}$ & 0.0071 \\
\hline$L C L$ filter & Grid-side reactance & $X_{l g}$ & 0.0735 \\
& Grid-side resistance & $R_{l g}$ & 0.0055 \\
& Converter-side reactance & $X_{l c}$ & 0.0808 \\
& Converter-side resistance & $R_{l c}$ & 0.0078 \\
& Capacitance reactance & $X_{c}$ & 0.0355 \\
& Capacitance resistance & $R_{c}$ & $0.0623 \cdot 10^{-3}$ \\
\hline Converter & Dc-link & $V_{\mathrm{dc}}$ & 1.9902 \\
\hline
\end{tabular}

Finally, a similar problem to (21) is solved, with the difference that the controller has to choose between two-instead of six - values of the objective function to determine the minimal one. In doing so, the optimal triplet $\left\{\boldsymbol{t}_{z}^{*}, \boldsymbol{U}_{z}^{*}, J_{z}^{*}\right\}$ is found, and, in line with the receding horizon policy, $\boldsymbol{u}_{a b c}^{*}\left(t_{1}^{*}(k)\right)$ and $\boldsymbol{u}_{a b c}^{*}\left(t_{2}^{*}(k)\right)$ are applied to the converter at the appropriate time instants.

The pseudocode of the proposed direct MPC with discontinuous modulation is presented in Algorithm 2. The block diagram is similar to that shown in Fig. 8, with the difference that the angle $\angle \boldsymbol{v}_{\text {conv,ref }}(k)$ needs to be fed into the MPC block.

\section{Performance Evaluation}

In this section the performance of the proposed direct MPC schemes is assessed on a simulation basis. The rated values as well as the p.u. parameters of the chosen case study (see Fig. 1) are given in Tables III and IV, respectively. The setup is assumed to be ideal, i.e., second-order effects, such as controller delays, deadtimes, measurement noise, parameter variations, model imperfections, disturbances, etc., are neglected. The chosen sampling interval is $T_{s}=175.43 \mu \mathrm{s}$. The weighting matrices for MPC with continuous and discontinuous modulation are chosen as $\boldsymbol{Q}=\operatorname{diag}(1,1,9,9,0.9,0.9)$ and $\boldsymbol{Q}=\operatorname{diag}(1,1,9,9,1.1,1.1)$, respectively, both prioritizing the grid current error. Moreover, matrix $\boldsymbol{\Lambda}$ for the two methods was chosen as $\boldsymbol{\Lambda}=\operatorname{diag}(9.5,9.5,10,10,10,10)$ and $\boldsymbol{\Lambda}=\operatorname{diag}(5.8,5.8,5.5,5.5,5.5,5.5)$, respectively. In the sequel, before presenting the performance evaluation, a brief description of the filter characteristics and the IEEE 519 grid standard is provided.

\section{A. LCL Filter}

The frequency response of the $L C L$ filter considered in this work is shown in Fig. 10. The filter has two resonance frequencies; the dominant one is given by (in SI)

$$
f_{\text {res }}=f_{B} \frac{1}{\sqrt{X_{c} \frac{X_{l_{c}\left(X_{l g}+X_{g}\right)}}{X_{l c}+X_{l_{g}}+X_{g}}}} \approx 1203.3 \mathrm{~Hz},
$$

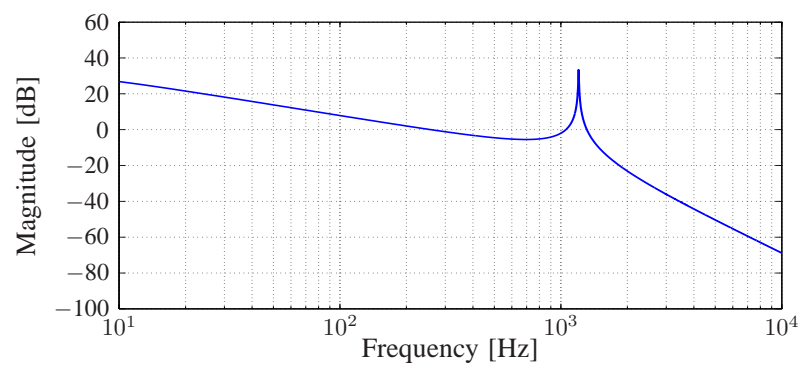

(a) Bode magnitude plot

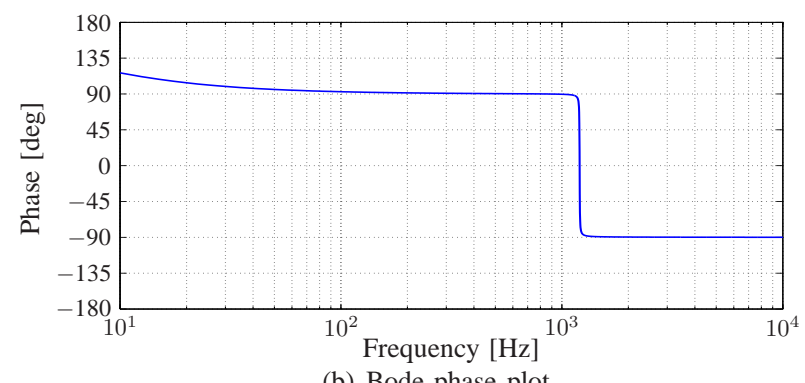

(b) Bode phase plot

Fig. 10: Frequency response of the $L C L$ filter.

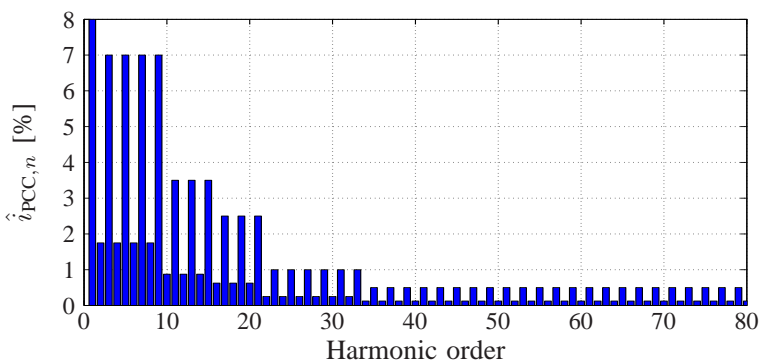

Fig. 11: Current harmonic limits at the PCC based on the IEEE 519 standard for short circuit ratio $k_{\mathrm{sc}}=20$.

where $f_{B}=50 \mathrm{~Hz}$ is the base (rated) frequency. As seen in the figure, $L C L$ filters-being third-order systems-can attenuate the harmonics beyond their resonance frequency at a rate of $-60 \mathrm{~dB} / \mathrm{dec}$. Nonetheless, the harmonics around the dominant resonance frequency are usually excited due to the low impedance of the filter at the vicinity of $f_{\text {res }}$. Thus, passive and/or active damping techniques are often used to provide sufficient damping of the filter resonance [45], [46].

\section{B. IEEE 519 Grid Code}

Grid codes are a set of regulations that define minimum requirements for the interconnection of facilities to the grid in order to ensure safe and secure operation of the system. The IEEE 519 grid standard [35] imposes limits on the current harmonics at the PCC. The latter is "usually taken as the point in the power system closest to the user where the system owner or operator could offer services to another user" [35] and is shown in Fig. 1.

For systems rated between $120 \mathrm{~V}$ and $69 \mathrm{kV}$, such as this case study, the maximum current harmonic limits at PCC are given in [35] as a percentage of the maximum fundamental frequency component of the current. The limits vary for different short-circuit ratios of the grid $k_{\mathrm{sc}}$. For $k_{\mathrm{sc}}=20$ considered in this work, the limits are depicted in Fig. 11. As 


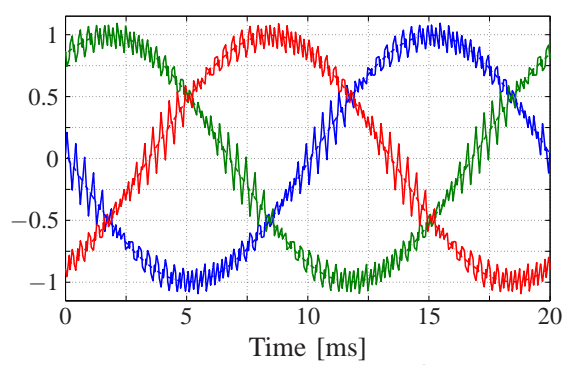

(a) Three-phase converter currents $\boldsymbol{i}_{\text {conv, } a b c}$ lines) and their references (dash-dotted lines).

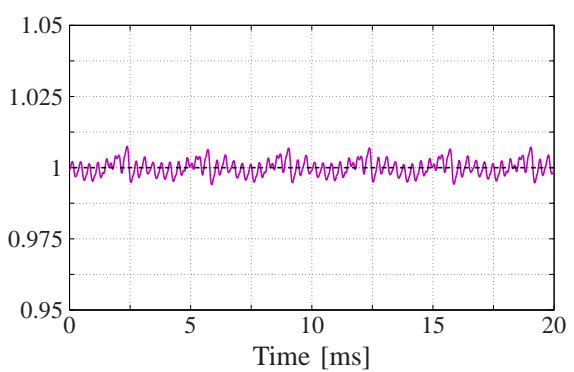

(d) Real power $P$ (dash-dotted line).

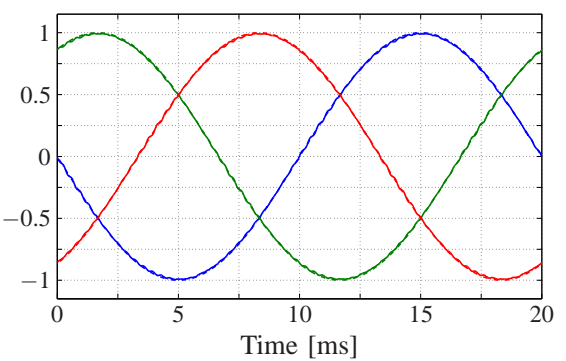

(b) Three-phase grid currents $i_{g, a b c}$ and their references (dash-dotted lines)

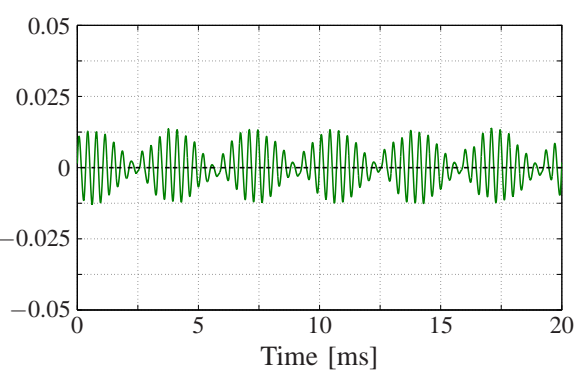

(e) Reactive power $Q$ (solid line) and its reference (dash-dotted line).

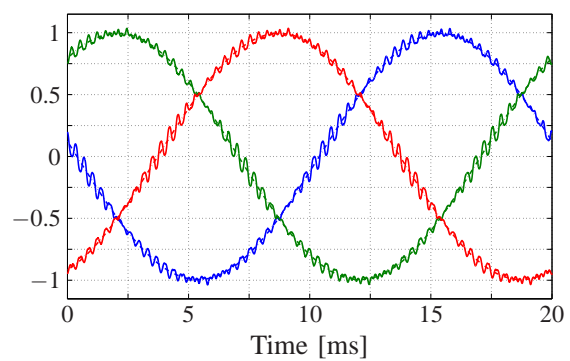

(c) Three-phase capacitor voltages $\boldsymbol{v}_{c, a b c}$ (solid lines) and their references (dash-dotted lines).

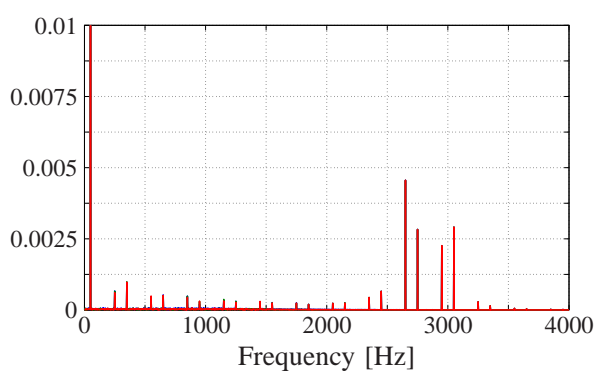

(f) Grid current spectrum. The TDD is $0.69 \%$.

Fig. 12: Simulated waveforms produced by direct MPC with continuous modulation during steady-state operation and unity power factor $\left(f_{\mathrm{sw}}=2850 \mathrm{~Hz}\right)$.

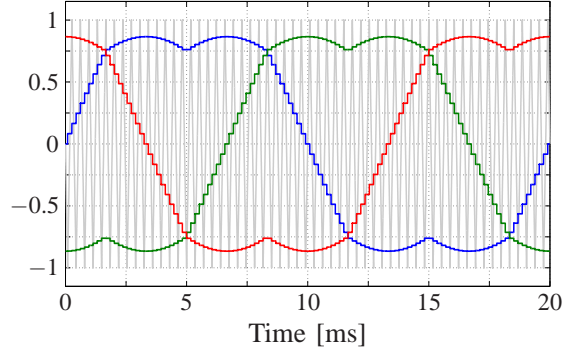

(a) Three-phase modulating signal and triangular carrier.

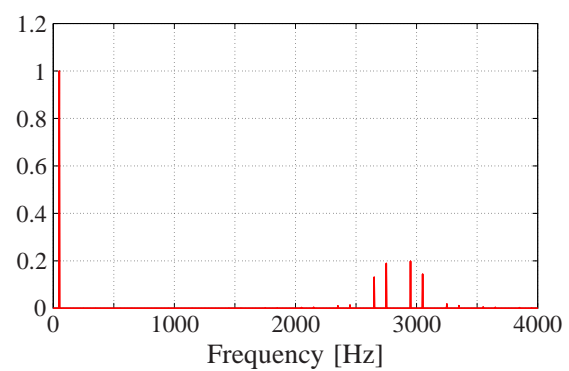

(b) Harmonic spectrum of differential-mode converter voltage.

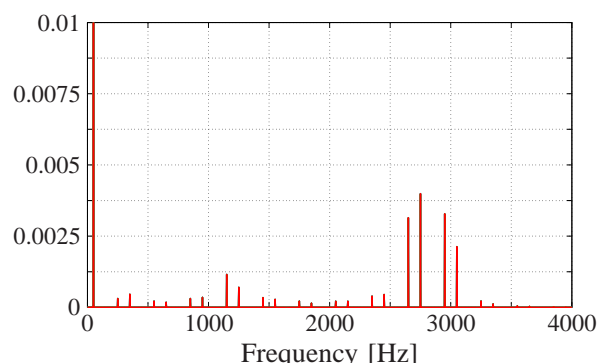

(c) Grid current spectrum. The TDD is $0.67 \%$.

Fig. 13: Simulated waveforms produced by asymmetric regularly sampled CB-PWM with min/max common-mode signal injection $\left(f_{\mathrm{sw}}=2850 \mathrm{~Hz}\right)$.

can be seen, the limits on even and higher-order harmonics are particularly stringent.

\section{Direct MPC with Continuous Modulation}

The closed-loop behavior of the system is examined while being controlled with the proposed direct MPC scheme with continuous modulation. With the chosen sampling interval of $T_{s}=175.43 \mu \mathrm{s}$ a fixed switching frequency of $f_{\mathrm{sw}}=2850 \mathrm{~Hz}$ results. The steady-state system performance when operating under unity power factor (pf), i.e., $P=1$ p.u. and $Q=0$ p.u., is shown in Fig. 12 for one fundamental period. The controlled variables track their reference values accurately, as can be seen in Figs. 12(a)-12(c), while operation under unity power factor is achieved (see Figs. 12(d) and 12(e)). The grid current TDD is as low as $0.69 \%$, thanks to the effective attenuation of the harmonics achieved by MPC with the chosen objective function (16) and the $L C L$ filter. Moreover, as can be seen in Fig. 12(f), where the grid current harmonic spectrum is shown, its harmonics appear at odd non-triplen multiples of the fundamental frequency with the more pronounced being at the vicinity of the switching frequency. Comparing with the harmonic limitations imposed by the IEEE 519 standard (see Fig. 11), it can be concluded that the produced grid current does not violate them. Furthermore, it is observed, that the harmonics in the vicinity of the resonance frequency are not excited despite the absence of an active damping loop and the low ratio $f_{\mathrm{sw}} / f_{\text {res }}$, which is $\approx 2.37$. Therefore, the proposed method can operate the converter at relatively low switching frequencies, and, thus, the switching losses can be reduced.

For comparison purposes, asymmetric regularly sampled CB-PWM with $\min / \max$ common-mode signal injection is implemented. Note that this leads to equivalence between CBPWM and SVM [34, Section 6.3]. Such a method can be interpreted as a closed-loop linear controller with a very low bandwidth. As before, nonidealities and second-order effects that appear in a real-world setting are neglected. Thus, the steady-state performance of this benchmark case corresponds to its theoretically best achievable performance. Moreover, owing to the harmonic energy distribution of SVM, lower harmonic distortion levels can be achieved while exploiting an extended linear modulation range [34]. Finally, for a fair and meaningful comparison, the carrier signal has the same 


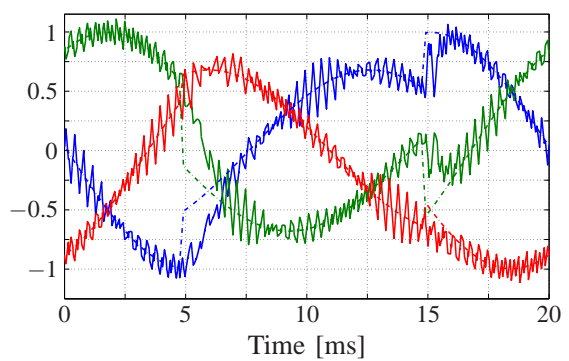

(a) Three-phase converter currents $\boldsymbol{i}_{\text {conv, } a b c}$ ( lines) and their references (dash-dotted lines).

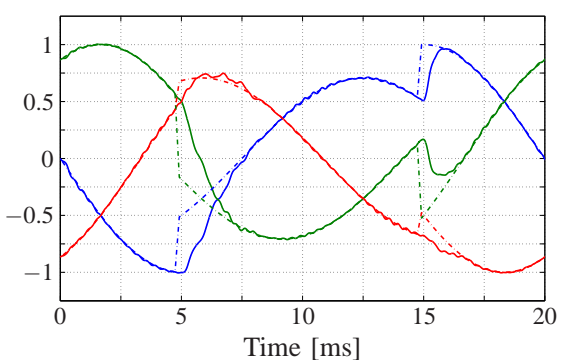

(b) Three-phase grid currents $\boldsymbol{i}_{g, a b c}$ (solid lines) and their references (dash-dotted lines).

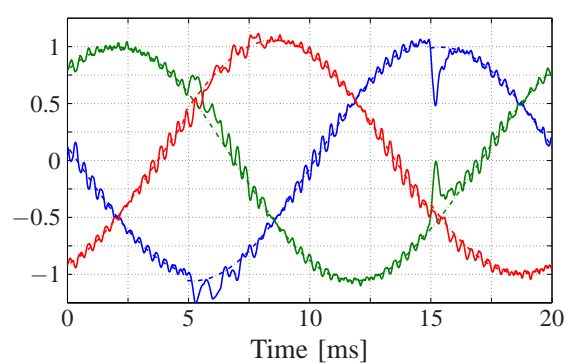

(c) Three-phase capacitor voltages $\boldsymbol{v}_{c, a b c}$ (solid lines) and their references (dash-dotted lines).

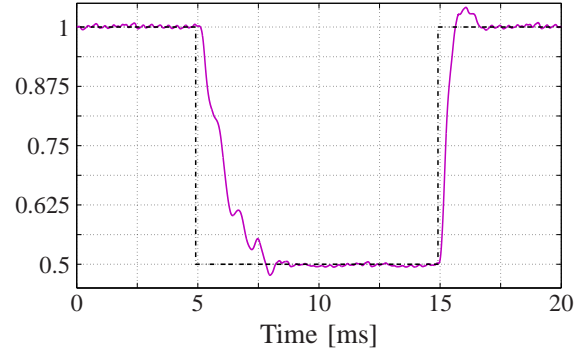

(d) Real power $P$ (solid line) and its reference (dash-dotted line).

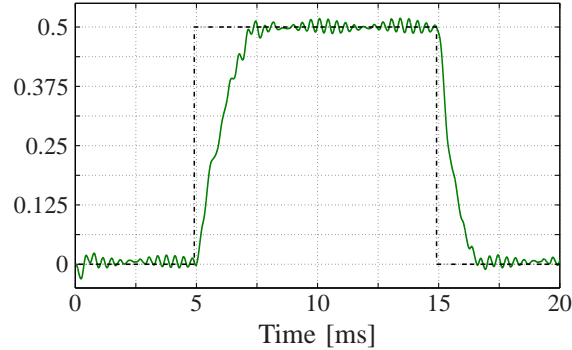

(e) Reactive power $Q$ (solid line) and its reference (dash-dotted line).

Fig. 14: Power reference steps for direct MPC with continuous modulation.

frequency as the switching frequency of MPC, i.e., $2850 \mathrm{~Hz}$.

The three-phase modulating signal and the triangular carrier waveform are shown in Fig. 13(a). Moreover, Figs. 13(b) and 13(c) illustrate the harmonic spectrum of the converter (differential-mode) output voltage and grid current, respectively. As seen in Fig. 13(c), the grid current harmonics appear at the same frequencies, but they are of different amplitudes compared with those produced by MPC (see Fig. 12(f)). The TDD of the grid current is $0.67 \%$. Hence, the proposed direct MPC achieves a grid current TDD which is almost equal to that produced by SVM.

Finally, the transient behavior of the proposed direct MPC with continuous modulation is examined during changes in real and reactive power references. At $t=5 \mathrm{~ms}$, both real and reactive power references are changed from the nominal operating point ( $P=1$ and $Q=0$ p.u.) to 0.5 p.u., and changed back at $t=15 \mathrm{~ms}$ to regain nominal operation. These steps on the power references are translated into the corresponding steady-state references of the controlled variables $\boldsymbol{y}$, i.e., the converter and grid currents, and capacitor voltage.

During power transients, there is an energy exchange between the converter, filter and grid. As a result, the magnitude and phase of the converter and grid current are changed. The same applies to the phase of the capacitor voltage. Despite all these changes, the controlled variables, and consequently the powers, track their references accurately with short settling times, see Fig. 14. Since the proposed MPC algorithm is implemented as a direct controller, it makes decisions such that the deviation of the controlled variables from their references is alleviated as quickly as possible. In effect, the dynamic response of MPC is limited by the available voltage margin. For example, due to the smaller voltage margin available when operating under unity pf, the dynamics are slower during the step-down-compared to the step-up—change in the real power.

\section{Direct MPC with Discontinuous Modulation}

When direct MPC emulates DPWMMIN (see Section IV) and the same sampling interval is used, i.e., $T_{s}=175.43 \mu \mathrm{s}$, then the resulting switching frequency is two-thirds of that in Section V-C. Therefore, the switching frequency for MPC with discontinuous modulation is $f_{\mathrm{sw}}=\frac{2}{3} \cdot 2850=1900 \mathrm{~Hz}$. The steady-state performance of the system at the same operating point as in Section V-C, i.e., $P=1$ and $Q=0$ p.u., is examined. The results are shown in Fig. 15. As can be seen in Figs. 15(d) and 15(e), operation under unity power factor is achieved. This implies, that the controlled variables accurately track their desired values (Figs. 15(a)-15(c)), albeit with slightly higher ripple due to the lower switching frequency. The increased ripples are also reflected in the grid current harmonic spectrum, see Fig. 15(f). As expected, the grid current TDD is slightly increased to $0.87 \%$. As can be seen in Fig. 15(f), harmonic energy is distributed not only among odd non-triplen multiples of the fundamental frequency, but also over even harmonics, since quarter- and half-wave symmetries are compromised with discontinuous modulation. Notwithstanding the foregoing, the produced grid current harmonics abide by the limitations imposed by the IEEE 519 standard. Furthermore, despite the ratio between the switching and resonance frequency being $\approx 1.58$, i.e., considerably low, the harmonics around the resonance frequency are not excited. Therefore, as can be seen, the proposed direct MPC, thanks to the full-state information (see (5) or (7)) and the refinements discussed in Section III-B (e.g., the long horizon [38]), effectively mitigates the adverse effect of the filter resonance, rendering an additional damping loop unnecessary. Thus, MPC with discontinuous modulation can operate the converter at even lower switching frequencies compared with MPC and 


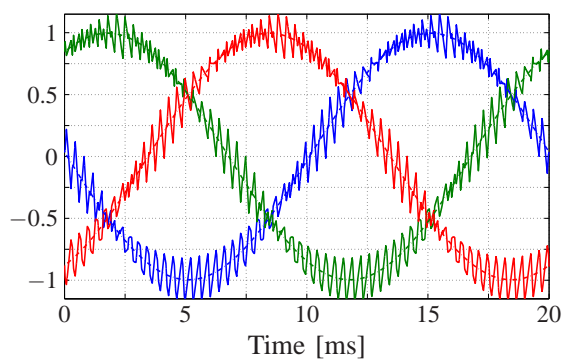

(a) Three-phase converter currents $\boldsymbol{i}_{\text {conv, } a b c}$ (sol lines) and their references (dash-dotted lines).

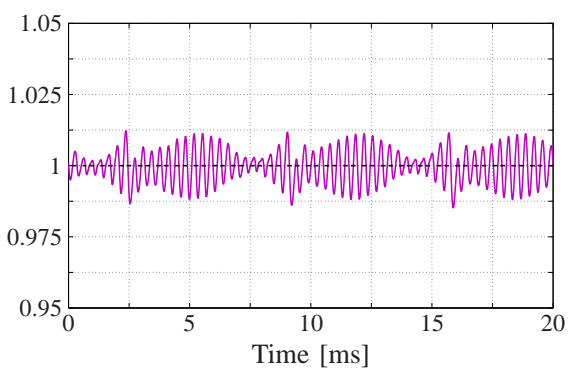

(d) Real power $P$ (dash-dotted line).

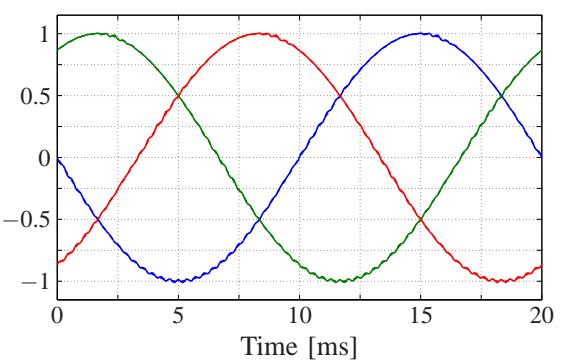

(b) Three-phase grid currents $\boldsymbol{i}_{g, a b c}$ (solid lines) and their references (dash-dotted lines).

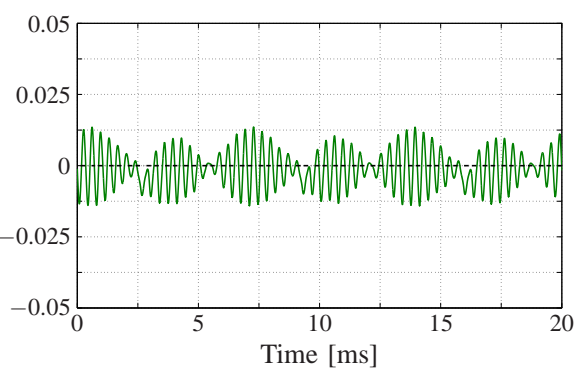

(e) Reactive power $Q$ (solid line) and its reference (dash-dotted line).

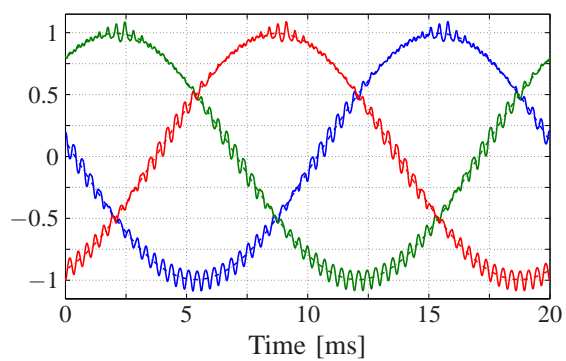

(c) Three-phase capacitor voltages $\boldsymbol{v}_{c, a b c}$ (solid lines) and their references (dash-dotted lines).

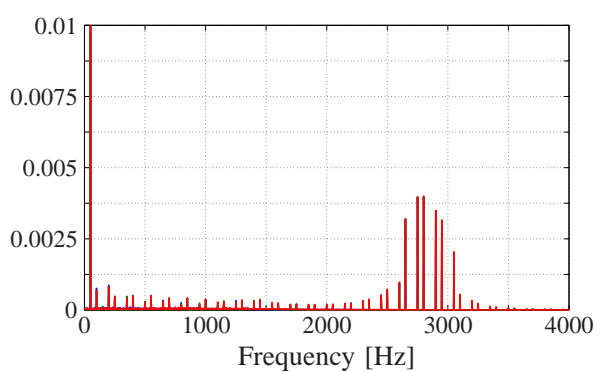

(f) Grid current spectrum. The TDD is $0.87 \%$.

Fig. 15: Simulated waveforms produced by direct MPC with discontinuous modulation during steady-state operation and unity power factor $\left(f_{\mathrm{sw}}=1900 \mathrm{~Hz}\right)$

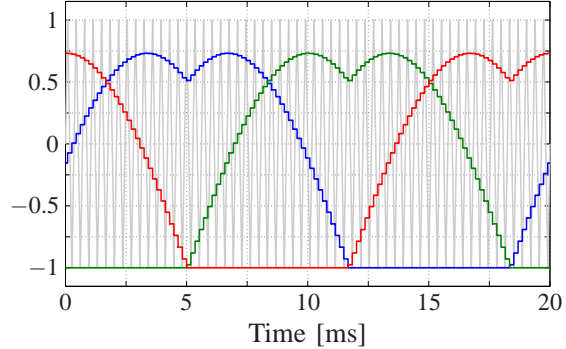

(a) Three-phase modulating signal and triangular carrier.

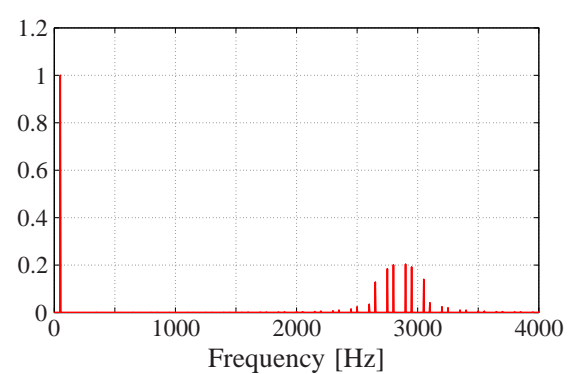

(b) Harmonic spectrum of differential-mode converter voltage.

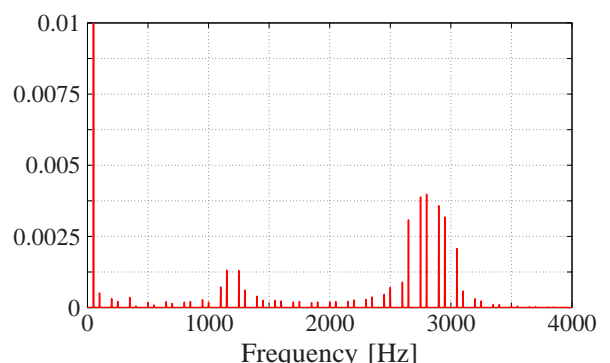

(c) Grid current spectrum. The TDD is $0.87 \%$.

Fig. 16: Simulated waveforms produced by asymmetric regularly sampled DPWMMIN $\left(f_{\mathrm{sw}}=1900 \mathrm{~Hz}\right)$.

continuous modulation. As a result, the switching power losses can be further reduced.

As a benchmark, asymmetric regularly sampled DPWMMIN is implemented for operation at the same switching frequency, i.e., $f_{\mathrm{sw}}=1900 \mathrm{~Hz}$. Fig. 16(a) illustrates the three-phase modulating signal and the triangular carrier waveform, while Figs. 16(b) and 16(c) show the spectrum of the converter (differential-mode) output voltage and grid current, respectively. As can be seen in Fig. 16(c), the harmonics produced by DPWMMIN are of the same order, but slightly different amplitude, compared with those produced by direct MPC. Despite these small differences, the grid current TDD is $0.87 \%$, i.e., equal to the one obtained with the proposed method.

As a last performance assessment, the dynamic behavior of the proposed scheme is examined during step changes in the power references, see Fig. 17. While operating at nominal operating conditions $(\mathrm{pf}=1)$, the real and reactive power references are changed to 0.5 p.u. at $t=5 \mathrm{~ms}$, and changed back to their values that correspond to nominal operation at $t=15 \mathrm{~ms}$. The controlled variables - and hence the powers- follow their references accurately. Since only two-rather than three-phases at a time are involved in switching, the transients are slightly slower compared with MPC with continuous modulation, see Section V-C. Nevertheless, the settling times are as short as possible, limited only by the available voltage margin.

\section{CONClusions}

This paper presents a direct MPC strategy for a threephase two-level grid-connected converter with an $L C L$ filter. In contrast to conventional direct MPC (FCS-MPC) algorithms, where the harmonic spectra are non-deterministic, with the harmonic energy spread over the whole range of frequencies, the proposed controller produces a discrete grid current harmonic spectrum. This is achieved by introducing a fixed modulation cycle and symmetrical switching patterns computed with the presented optimization problem underlying direct MPC. In doing so, the grid standards-such as IEEE 519 - can be met since the stringent limits on harmonics, especially of even order and interharmonics, are adhered to, while the grid current TDD is very close to that of asymmetric 


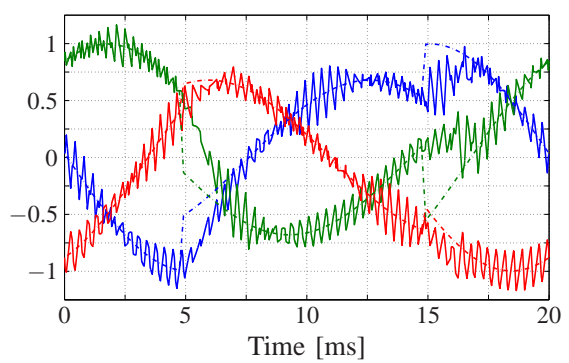

(a) Three-phase converter currents $\boldsymbol{i}_{\text {conv, } a b c}$ ( lines) and their references (dash-dotted lines).

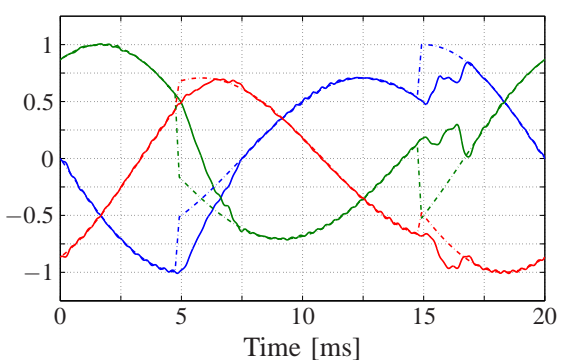

(b) Three-phase grid currents $\boldsymbol{i}_{g, a b c}$ (solid lines) and their references (dash-dotted lines).

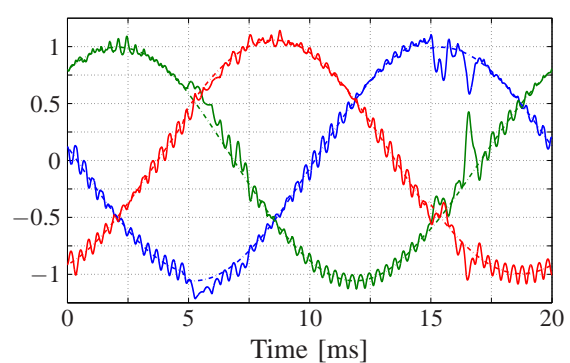

c) Three-phase capacitor voltages $\boldsymbol{v}_{c, a b c}$ (solic lines) and their references (dash-dotted lines).

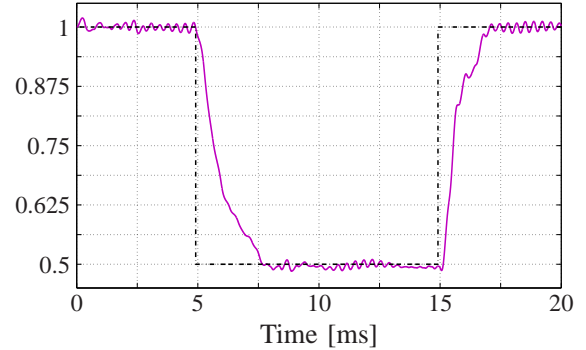

(d) Real power $P$ (solid line) and its reference (dash-dotted line).

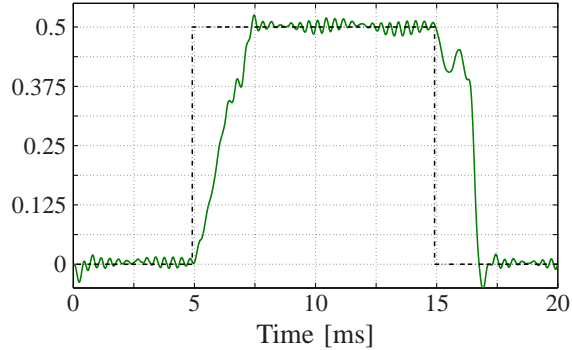

(e) Reactive power $Q$ (solid line) and its reference (dash-dotted line).

Fig. 17: Power reference steps for direct MPC with discontinuous modulation.

regularly sampled CB-PWM with $\min / \max$ common-mode signal injection (i.e., SVM). Moreover, by exploiting the inherent full-state-control mechanism of MPC, an additional active damping loop is redundant. Furthermore, owing to the adopted MIMO approach, MPC manages to successfully control all output variables not only at steady-state operating conditions, but also during transients. As a result, the fast transient responses that characterize direct control schemes, as the proposed one, are still present.

In an attempt to further reduce the switching frequency of the converter, the algorithm is modified to emulate the behavior of $120^{\circ}$ discontinuous PWM. By doing so, the switching frequency is reduced by $33 \%$ compared with MPC with continuous modulation. Moreover, albeit the lower switching frequency, the grid current harmonics still adhere to the grid codes, while the grid current TDD is the same as the one achieved with asymmetric regularly sampled DPWMMIN. Further, it is shown that even when a discontinuous switching pattern is implemented by MPC the dynamic behavior of the system does not deteriorate.

It can be concluded, that both of the proposed direct MPC approaches can operate the system even when the ratio between the switching frequency and the resonance frequency is as small as two. This is thanks to the high bandwidth of the controller(s), which is higher than that of indirect control methods, such as vector control with SVM/DPWM. Therefore, owing to the proposed direct MPC approach, one can increase the efficiency of the converter by reducing the switching frequency, and, consequently, the switching losses, while the harmonic output spectrum meets the grid standards, e.g., [35]. Alternatively, one can reduce the size of the $L C L$ filter by increasing the frequency of the filter resonance.

\section{APPENDIX}

Vector $\boldsymbol{r}$ and matrix $\boldsymbol{M}$ in (18) are given by

$$
\boldsymbol{r}=\left[\begin{array}{c}
\boldsymbol{y}_{\mathrm{ref}}\left(t_{0}\right)-\boldsymbol{y}\left(t_{0}\right) \\
\boldsymbol{y}_{\mathrm{ref}}\left(t_{0}\right)-\boldsymbol{y}\left(t_{0}\right) \\
\boldsymbol{y}_{\mathrm{ref}}\left(t_{0}\right)-\boldsymbol{y}\left(t_{0}\right) \\
\boldsymbol{\Lambda}\left(\boldsymbol{y}_{\mathrm{ref}}\left(T_{s}\right)-\boldsymbol{y}\left(t_{0}\right)-\boldsymbol{m}\left(t_{3}(k)\right) T_{s}\right) \\
\boldsymbol{y}_{\mathrm{ref}}\left(T_{s}\right)-\boldsymbol{y}\left(t_{0}\right) \\
\boldsymbol{y}_{\mathrm{ref}}\left(T_{s}\right)-\boldsymbol{y}\left(t_{0}\right) \\
\boldsymbol{y}_{\mathrm{ref}}\left(T_{s}\right)-\boldsymbol{y}\left(t_{0}\right) \\
\boldsymbol{\Lambda}\left(\boldsymbol{y}_{\mathrm{ref}}\left(2 T_{s}\right)-\boldsymbol{y}\left(t_{0}\right)-\boldsymbol{m}\left(t_{3}(k+1)\right) 2 T_{s}\right)
\end{array}\right],
$$

and

$$
\boldsymbol{M}=\left[\begin{array}{cccccc}
\boldsymbol{m}_{t_{0}} & \mathbf{0}_{6} & \mathbf{0}_{6} & \mathbf{0}_{6} & \mathbf{0}_{6} & \mathbf{0}_{6} \\
\boldsymbol{m}_{0} & \boldsymbol{m}_{t_{1}} & \mathbf{0}_{6} & \mathbf{0}_{6} & \mathbf{0}_{6} & \mathbf{0}_{6} \\
\boldsymbol{m}_{0} & \boldsymbol{m}_{1} & \boldsymbol{m}_{t_{2}} & \mathbf{0}_{6} & \mathbf{0}_{6} & \mathbf{0}_{6} \\
\boldsymbol{\Lambda} \boldsymbol{m}_{0} & \boldsymbol{\Lambda} \boldsymbol{m}_{1} & \boldsymbol{\Lambda} \boldsymbol{m}_{2} & \mathbf{0}_{6} & \mathbf{0}_{6} & \mathbf{0}_{6} \\
\boldsymbol{m}_{0} & \boldsymbol{m}_{1} & \boldsymbol{m}_{2} & \widetilde{\boldsymbol{m}}_{t_{0}} & \mathbf{0}_{6} & \mathbf{0}_{6} \\
\boldsymbol{m}_{0} & \boldsymbol{m}_{1} & \boldsymbol{m}_{2} & \widetilde{\boldsymbol{m}}_{0} & \widetilde{\boldsymbol{m}}_{t_{1}} & \mathbf{0}_{6} \\
\boldsymbol{m}_{0} & \boldsymbol{m}_{1} & \boldsymbol{m}_{2} & \widetilde{\boldsymbol{m}}_{0} & \widetilde{\boldsymbol{m}}_{1} & \widetilde{\boldsymbol{m}}_{t_{2}} \\
\boldsymbol{\Lambda} \boldsymbol{m}_{0} & \boldsymbol{\Lambda} \boldsymbol{m}_{1} & \boldsymbol{\Lambda} \boldsymbol{m}_{2} & \boldsymbol{\Lambda} \widetilde{\boldsymbol{m}}_{0} & \boldsymbol{\Lambda} \widetilde{\boldsymbol{m}}_{1} & \boldsymbol{\Lambda} \widetilde{\boldsymbol{m}}_{2}
\end{array}\right]
$$

with

$$
\begin{aligned}
\boldsymbol{m}_{t_{i}} & =\boldsymbol{m}\left(t_{i}(k)\right)-\boldsymbol{m}_{\mathrm{ref}}(k), \\
\widetilde{\boldsymbol{m}}_{t_{i}} & =\boldsymbol{m}\left(t_{i}(k+1)\right)-\boldsymbol{m}_{\mathrm{ref}}(k+1), \\
\boldsymbol{m}_{i} & =\boldsymbol{m}\left(t_{i}(k)\right)-\boldsymbol{m}\left(t_{i+1}(k)\right), \\
\widetilde{\boldsymbol{m}}_{i} & =\boldsymbol{m}\left(t_{i}(k+1)\right)-\boldsymbol{m}\left(t_{i+1}(k+1)\right),
\end{aligned}
$$

where $i \in\{0,1,2\}$, and $t_{0}(k+1)=T_{s}$. 


\section{ACKNOWLEDGMENT}

M. Nahalparvari would like to thank ABB Oy Drives, Helsinki, Finland, for the financial support.

\section{REFERENCES}

[1] J. B. Rawlings and D. Q. Mayne, Model predictive control: Theory and design. Madison, WI: Nob Hill, 2009.

[2] J. Holtz and S. Stadtfeld, "A predictive controller for the stator current vector of ac-machines fed from a switched voltage source," in Int. Power Electron. Conf., Tokyo, Japan, Mar. 1983, pp. 1665-1675.

[3] P. Cortés, M. P. Kazmierkowski, R. M. Kennel, D. E. Quevedo, and J. Rodríguez, "Predictive control in power electronics and drives," IEEE Trans. Ind. Electron., vol. 55, no. 12, pp. 4312-4324, Dec. 2008.

[4] T. Geyer, G. Papafotiou, and M. Morari, "Hybrid model predictive control of the step-down dc-dc converter," IEEE Trans. Control Syst. Technol., vol. 16, no. 6, pp. 1112-1124, Nov. 2008

[5] S. Mariéthoz and M. Morari, "Explicit model-predictive control of a PWM inverter with an $L C L$ filter," IEEE Trans. Ind. Electron., vol. 56, no. 2, pp. 389-399, Feb. 2009.

[6] S. Almér, S. Mariéthoz, and M. Morari, "Sampled data model predictive control of a voltage source inverter for reduced harmonic distortion," IEEE Trans. Control Syst. Technol., vol. 21, no. 5, pp. 1907-1915, Sep. 2013.

[7] A. G. Beccuti, G. Papafotiou, and L. Harnefors, "Multivariable optimal control of HVDC transmission links with network parameter estimation for weak grids," IEEE Trans. Control Syst. Technol., vol. 22, no. 2, pp. 676-689, Mar. 2014.

[8] T. Geyer, Model predictive control of high power converters and industrial drives. Hoboken, NJ: Wiley, 2016.

[9] L. A. Wolsey, Integer programming. New York, NY: Wiley, 1998.

[10] T. Geyer and D. E. Quevedo, "Multistep finite control set model predictive control for power electronics," IEEE Trans. Power Electron., vol. 29 , no. 12 , pp. $6836-6846$, Dec. 2014.

[11] P. Karamanakos, T. Geyer, and R. Kennel, "A computationally efficient model predictive control strategy for linear systems with integer inputs," IEEE Trans. Control Syst. Technol., vol. 24, no. 4, pp. 1463-1471, Jul. 2016.

[12] J. Rodríguez, J. Pontt, C. A. Silva, P. Correa, P. Lezana, P. Cortés, and U. Ammann, "Predictive current control of a voltage source inverter," IEEE Trans. Ind. Electron., vol. 54, no. 1, pp. 495-503, Feb. 2007.

[13] J. Rodríguez and P. Cortés, Predictive control of power converters and electrical drives. Chichester, UK: Wiley, 2012.

[14] T. Geyer, "A comparison of control and modulation schemes for medium-voltage drives: Emerging predictive control concepts versus PWM-based schemes," IEEE Trans. Ind. Appl., vol. 47, no. 3, pp. 13801389, May/Jun. 2011.

[15] P. Karamanakos and T. Geyer, "Guidelines for the design of finite control set model predictive controllers," IEEE Trans. Power Electron., vol. 35, no. 7, pp. 7434-7450, Jul. 2020

[16] A. Bouafia, J.-P. Gaubert, and F. Krim, "Predictive direct power control of three-phase pulsewidth modulation (PWM) rectifier using spacevector modulation (SVM)," IEEE Trans. Power Electron., vol. 25, no. 1, pp. 228-236, Jan. 2010

[17] R. O. Ramírez, J. R. Espinoza, F. Villarroel, E. Maurelia, and M. E. Reyes, "A novel hybrid finite control set model predictive control scheme with reduced switching," IEEE Trans. Ind. Electron., vol. 61, no. 11, pp. 5912-5920, Nov. 2014

[18] P. Cortés, J. Rodríguez, D. E. Quevedo, and C. Silva, "Predictive current control strategy with imposed load current spectrum," IEEE Trans. Power Electron., vol. 23, no. 2, pp. 612-618, Mar. 2008.

[19] M. Pacas and J. Weber, "Predictive direct torque control for the PM synchronous machine," IEEE Trans. Ind. Electron., vol. 52, no. 5, pp. 1350-1356, Oct. 2005.

[20] Y. Zhang, W. Xie, Z. Li, and Y. Zhang, "Model predictive direct power control of a PWM rectifier with duty cycle optimization," IEEE Trans. Power Electron., vol. 28, no. 11, pp. 5343-5351, Nov. 2013.

[21] P. Karamanakos, P. Stolze, R. M. Kennel, S. Manias, and H. du T. Mouton, "Variable switching point predictive torque control of induction machines," IEEE J. Emerg. Sel. Topics Power Electron., vol. 2, no. 2, pp. 285-295, Jun. 2014

[22] Y. Zhang, W. Xie, Z. Li, and Y. Zhang, "Low-complexity model predictive power control: Double-vector-based approach," IEEE Trans. Ind. Electron., vol. 61, no. 11, pp. 5871-5880, Nov. 2014.
[23] L. Tarisciotti, P. Zanchetta, A. Watson, J. C. Clare, M. Degano, and S. Bifaretti, "Modulated model predictive control for a three-phase active rectifier," IEEE Trans. Ind. Appl., vol. 51, no. 2, pp. 1610-1620, Mar./Apr. 2015.

[24] Q. Liu and K. Hameyer, "Torque ripple minimization for direct torque control of PMSM with modified FCSMPC," IEEE Trans. Ind. Appl., vol. 52, no. 6, pp. 4855-4864, Nov./Dec. 2016.

[25] Y. Zhang, Y. Peng, and C. Qu, "Model predictive control and direct power control for PWM rectifiers with active power ripple minimization," IEEE Trans. Ind. Appl., vol. 52, no. 6, pp. 4909-4918, Nov./Dec. 2016.

[26] M. Tomlinson, H. du T. Mouton, R. Kennel, and P. Stolze, "A fixed switching frequency scheme for finite-control-set model predictive control-Concept and algorithm," IEEE Trans. Ind. Electron., vol. 63, no. 12, pp. 7662-7670, Dec. 2016.

[27] L. Tarisciotti, J. Lei, A. Formentini, A. Trentin, P. Zanchetta, P. Wheeler and M. Rivera, "Modulated predictive control for indirect matrix converter," IEEE Trans. Ind. Appl., vol. 53, no. 5, pp. 4644-4654, Sep./Oct. 2017.

[28] P. Karamanakos, A. Ayad, and R. Kennel, "A variable switching point predictive current control strategy for quasi-Z-source inverters," IEEE Trans. Ind. Appl., vol. 54, no. 2, pp. 1469-1480, Mar./Apr. 2018.

[29] S. Vazquez, A. Marquez, R. Aguilera, D. Quevedo, J. I. Leon, and L. G. Franquelo, "Predictive optimal switching sequence direct power control for grid-connected power converters," IEEE Trans. Ind. Electron., vol. 62, no. 4, pp. 2010-2020, Apr. 2015.

[30] S. A. Larrinaga, M. A. R. Vidal, E. Oyarbide, and J. R. T. Apraiz, "Predictive control strategy for dc/ac converters based on direct power control," IEEE Trans. Ind. Electron., vol. 54, no. 3, pp. 1261-1271, Jun. 2007.

[31] S. Vazquez, R. P. Aguilera, P. Acuna, J. Pou, J. I. Leon, L. G. Franquelo, and V. G. Agelidis, "Model predictive control for single-phase NPC converters based on optimal switching sequences," IEEE Trans. Ind. Electron., vol. 63, no. 12, pp. 7533-7541, Dec. 2016.

[32] W. Falmbigl, O. König, S. Jakubek, and G. Prochart, "Predictive pulse pattern control for a synchronous multiphase buck converter," in Proc IEEE Int. Symp. Pred. Control of Elect. Drives and Power Electron., Pilsen, Czech Republic, Sep. 2017, pp. 1-6.

[33] P. Karamanakos, R. Mattila, and T. Geyer, "Fixed switching frequency direct model predictive control based on output current gradients," in Proc. IEEE Ind. Electron. Conf., Washington, D.C., Oct. 2018, pp. 23292334.

[34] D. G. Holmes and T. A. Lipo, Pulse width modulation for power converters: Principles and practice. Piscataway, NJ: IEEE Press, 2003.

[35] IEEE Std 519-2014 (Revision of IEEE Std 519-1992), "IEEE recommended practices and requirements for harmonic control in electrical power systems," pp. 1-29, Jun. 2014.

[36] S. G. Parker, B. P. McGrath, and D. G. Holmes, "Regions of active damping control for $L C L$ filters," IEEE Trans. Ind. Appl., vol. 50, no. 1, pp. 424-432, Jan./Feb. 2014.

[37] S. N. Manias, Power electronics and motor drive systems. Cambridge, MA: Academic Press, 2016.

[38] T. Geyer, P. Karamanakos, and R. Kennel, "On the benefit of longhorizon direct model predictive control for drives with $L C$ filters," in Proc. IEEE Energy Convers. Congr. Expo., Pittsburgh, PA, Sep. 2014, pp. 3520-3527.

[39] S. Boyd and L. Vandenberghe, Convex optimization. Cambridge, UK: Cambridge Univ. Press, 2004.

[40] Y. Wang and S. Boyd, "Fast model predictive control using online optimization," IEEE Trans. Control Syst. Technol., vol. 18, no. 2, pp. 267-278, Mar. 2010.

[41] S. Richter, S. Mariéthoz, and M. Morari, "High-speed online MPC based on a fast gradient method applied to power converter control," in Proc. Am. Control Conf., Baltimore, MD, Jun./Jul. 2010, pp. 4737-4743.

[42] H. Peyrl, A. Zanarini, T. Besselmann, J. Liu, and M.-A. Boéchat, "Parallel implementations of the fast gradient method for high-speed MPC," Control Eng. Pract., vol. 33, pp. 22-34, Dec. 2014.

[43] S. Richter, T. Geyer, and M. Morari, "Resource-efficient gradient methods for model predictive pulse pattern control on an FPGA," IEEE Trans. Control Syst. Technol., vol. 25, no. 3, pp. 828-841, May 2017.

[44] A. M. Trzynadlowski and S. Legowski, "Minimum-loss vector PWM strategy for three-phase inverters," IEEE Trans. Power Electron., vol. 9 , no. 1, pp. 26-34, Jan. 1994.

[45] M. Liserre, F. Blaabjerg, and S. Hansen, "Design and control of an $L C L$-filter-based three-phase active rectifier," IEEE Trans. Ind. Appl., vol. 41, no. 5, pp. 1281-1291, Sep./Oct. 2005. 
[46] J. Dannehl, F. W. Fuchs, S. Hansen, and P. B. Thøgersen, "Investigation of active damping approaches for PI-based current control of gridconnected pulse width modulation converters with $L C L$ filters," IEEE Trans. Ind. Appl., vol. 46, no. 4, pp. 1509-1517, Jul./Aug. 2010.

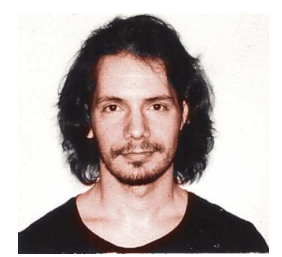

Petros Karamanakos (S'10-M'14-SM'19) received the Diploma and the Ph.D. degrees in electrical and computer engineering from the National Technical University of Athens (NTUA), Athens, Greece, in 2007, and 2013, respectively.

From 2010 to 2011 he was with the ABB Corporate Research Center, Baden-Dättwil, Switzerland, where he worked on model predictive control strategies for medium-voltage drives. From 2013 to 2016 he was a PostDoc Research Associate in the Chair of Electrical Drive Systems and Power Electronics, Technische Universität München, Munich, Germany. Since September 2016, he has been an Assistant Professor in the Faculty of Information Technology and Communication Sciences, Tampere University, Tampere, Finland. His main research interests lie at the intersection of optimal control, mathematical programming and power electronics, including model predictive control for power electronic converters and ac drives.

Dr. Karamanakos received the 2014 Third Best Paper Award of the IEEE Transactions on Industry Applications and the First Prize Paper Award of the Industrial Drives Committee at the 2013 IEEE Energy Conversion Congress and Exposition. He serves as an Associate Editor of the Industrial Power Converters Committee for the IEEE Transactions on Industry Applications.

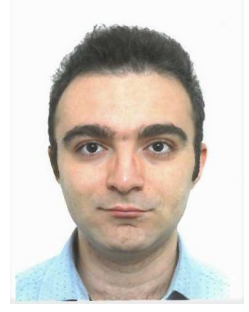

Mehrdad Nahalparvari (S'19) received the M.Sc. degree in power electronics from the Tampere University, Tampere, Finland, in 2019.

$\mathrm{He}$ is currently working towards the Ph.D. degree in electrical engineering at KTH Royal Institute of Technology, Stockholm, Sweden. His research interests include modeling and control of power electronic converters.

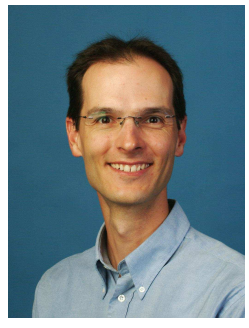

Tobias Geyer (M'08-SM'10) received the Dipl.Ing. and Ph.D. degrees in electrical engineering from ETH Zurich, Zurich, Switzerland, in 2000 and 2005, respectively, and the Habilitation degree in power electronics from ETH Zurich, Zurich, Switzerland, in 2017.

After his Ph.D., he spent three years at GE Global Research, Munich, Germany, three years at the University of Auckland, Auckland, New Zealand, and eight years at ABB's Corporate Research Centre, Baden-Dättwil, Switzerland. There, in 2016, he became a Senior Principal Scientist for power conversion control. He was appointed as an extraordinary Professor at Stellenbosch University, Stellenbosch, South Africa, from 2017 to 2020. In 2020, he joined ABB's medium-voltage drives business as R\&D platform manager of the ACS6000/6080.

$\mathrm{He}$ is the author of 35 patent applications and the book "Model predictive control of high power converters and industrial drives" (Wiley, 2016). $\mathrm{He}$ teaches a regular course on model predictive control at ETH Zurich. His research interests include medium-voltage and low-voltage drives, utility-scale power converters, optimized pulse patterns and model predictive control.

Dr. Geyer is the recipient of the 2017 First Place Prize Paper Award in the Transactions on Power Electronics, the 2014 Third Place Prize Paper Award in the Transactions on Industry Applications, and of two Prize Paper Awards at conferences. He is a former Associate Editor for the IEEE Transactions on Industry Applications (from 2011 until 2014) and the IEEE Transactions on Power Electronics (from 2013 until 2019). He was an international program committee vice chair of the IFAC conference on Nonlinear Model Predictive Control in Madison, WI, USA, in 2018. Dr. Geyer is a Distinguished Lecturer of the Power Electronics Society in the years 2020 and 2021. 